\title{
Cyclic LTI Systems in Digital Signal Processing
}

\author{
P. P. Vaidyanathan, Fellow, IEEE and Ahmet Kiraç, Student Member, IEEE
}

\begin{abstract}
Cyclic signal processing refers to situations where all the time indices are interpreted modulo some integer $L$. In such cases, the frequency domain is defined as a uniform discrete grid (as in $L$-point DFT). This offers more freedom in theoretical as well as design aspects. While circular convolution has been the centerpiece of many algorithms in signal processing for decades, such freedom, especially from the viewpoint of linear system theory, has not been studied in the past. In this paper, we introduce the fundamentals of cyclic multirate systems and filter banks, presenting several important differences between the cyclic and noncyclic cases. Cyclic systems with allpass and paraunitary properties are studied. The paraunitary interpolation problem is introduced, and it is shown that the interpolation does not always succeed. State-space descriptions of cyclic LTI systems are introduced, and the notions of reachability and observability of state equations are revisited. It is shown that unlike in traditional linear systems, these two notions are not related to the system minimality in a simple way. Throughout the paper, a number of open problems are pointed out from the perspective of the signal processor as well as the system theorist.
\end{abstract}

Index Terms - Cyclic systems, filterbanks-cyclic, waveletscyclic.

\section{INTRODUCTION}

C ONSIDER two sequences $x(n)$ and $h(n)$ defined for $0 \leq n \leq L-1$, and let $y(n)$ denote their circular or cyclic convolution [17]. That is, $y(n)=\Sigma_{m=0}^{L-1} x(m) h(n-m)$ with all arguments interpreted modulo $L$. We can represent this operation by the block diagram of Fig. 1, where $x(n)$ and $y(n)$ are the input and output, respectively, of a linear system. With all time-arguments interpreted modulo- $L$, this is also a time-invariant system (i.e., a circular-shift invariant system). We say that $\{h(n)\}$ is a cyclic LTI system. For the purpose of interpretation, we can also regard $x(n)$ to be a periodic$L$ input for which the LTI system $h(n)$ yields the periodic- $L$ output $y(n)$.

Circular or cyclic convolution has been at the center of digital signal processing from its early days [8]. Indeed, fast algorithms for ordinary convolution routinely convert the problem into a cyclic convolution and then use the FFT. The notion of polynomial transforms introduced by Nussbaumer [16] is another example of the prevalence of these ideas in the early days of digital signal processing. Most of the classical applications involving cyclic convolutions are based

Manuscript received November 13, 1997; revised August 6, 1998. This work supported in part by the Office of Naval Research under Grant N0001493-1-0231 and Tektronix, Inc. The associate editor coordinating the review of this paper and approving it for publication was Dr. Sergios Theodoridis.

P. P. Vaidyanathan is with the Department of Electrical Engineering, California Institute of Technology, Pasadena, CA 91125 USA.

A. Kirac is with Lucent Technologies, Huntington Beach, CA 92646 USA. Publisher Item Identifier S 1053-587X(99)00757-6.

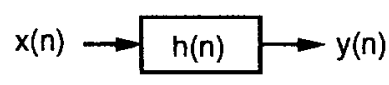

Fig. 1. Cyclic LTI system.

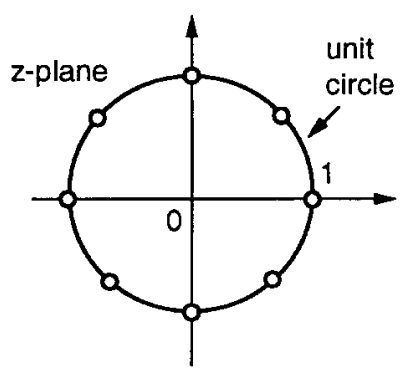

Fig. 2. Points on the unit-circle corresponding to the DFT frequencies $(L=8)$.

on the important result that cyclic convolution corresponds to multiplication of the DFT coefficients, that is, $Y(k)=$ $H(k) X(k)$ for $0 \leq k \leq L-1$. Further properties of cyclic LTI systems from the viewpoint of multirate signal processing (more generally linear system theory) have not been studied in the past. In this paper, we will consider a number of such properties. The emphasis will be on well-known topics [1], [12], [23], [27] such as multirate systems, filter banks, paraunitary matrices, and state-space representations but, this time, in a cyclic setting.

\section{A. Motivation and Scope}

The frequency response of a cyclic LTI system is the $L$-point DFT of the impulse response

$$
H(k)=\sum_{n=0}^{L-1} h(n) W_{L}^{k n}, \quad 0 \leq k \leq L-1
$$

where $W_{L}=e^{-j 2 \pi / L}$. This is equivalent to sampling the conventional frequency response $\Sigma_{n=0}^{L-1} h(n) e^{-j \omega n}$ at $L$ discrete values of the frequency $\omega_{k}=e^{j 2 \pi k / L}$ (the DFT-frequencies; see Fig. 2). The basic building blocks in the implementation of this system are multipliers, adders, and cyclic delays $W_{L}^{k}$. The cyclic delay, indicated in Fig. 3, has input-output relation $y(n)=x(n-1)$, where the time-arguments are interpreted modulo- $L$. Fig. 4 shows the direct-form structure for $H(k)$ using these building blocks.

In cyclic signal processing, the frequency domain is defined as a set of $L$ discrete frequencies rather than the entire range $0 \leq \omega<2 \pi$. This offers more freedom in theoretical developments. For example, we will see in Section IV that the definitions of allpass filters and paraunitary matrices are 


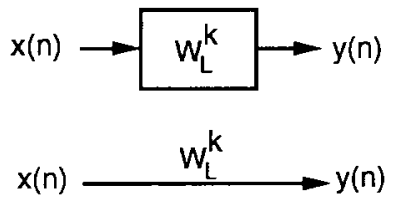

Fig. 3. Two ways to represent a $\operatorname{cyclic}(L)$ delay.

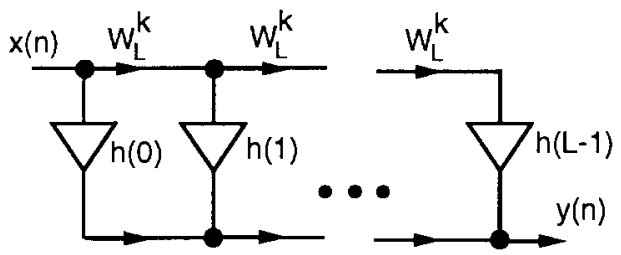

Fig. 4. Direct-form implementation of an arbitrary cyclic $(L)$ LTI system.

less restricted in the cyclic world. Similarly, orthonormal filter banks in the cyclic world are less restrictive. In subband and transform coding problems, if the power spectrum of the input signal is defined only over a discrete grid of frequencies and the filters optimized for these frequencies, it might offer an increased coding efficiency. Likewise, in filter design problems, one traditionally imposes constraints (e.g., linearity of phase) and optimizes the filter with respect to some criterion (e.g., minimax, minimum error energy, etc.). These constraints are now only over a discrete grid rather than a continuous range of frequencies. These are some of the motivations for considering cyclic signal processing as a theoretical discipline by itself. The practical advantages obtained using this viewpoint can be significant, but it requires further detailed work to quantify these advantages for specific applications. A unique example of the use of cyclic filter banks (brought to our attention by one of the reviewers) is the method of autoregressive spectral estimation in subbands, which was advanced by Nishikawa et al. in 1993 [14]. Our emphasis in this paper will only be on the theoretical differences between cyclic and noncyclic systems, especially pertaining to multirate systems, filter banks, and LTI system theory.

Related Literature: Since circular convolution is an integral part of DSP, we can regard cyclic LTI filtering as one of the earliest known DSP techniques [8]. The use of circular filtering in image subband coding (where images have finite size [7]) was motivated by the work of Smith and Eddins on symmetric extension techniques [19]. Circular convolution for subband coding was also explicitly considered by Kiya et al. [11]. In 1993, Caire et al. [5] introduced wavelet transforms associated with cyclic groups. Cyclic versions of two-channel filter-bank orthonormality and power complementarity can already be found in that paper. More recently, cylic multirate system basics were explicitly formulated in two independent conference papers [3], [24]. While both papers start with a common basic theme, the work by Bopardikar et al. [3], [4] eventually focuses on the wavelet aspect, whereas [24] focuses on system theoretic aspects, factorizations, and so forth. The very recent work in [13] focuses on interesting details of the two-channel linear phase orthonormal case. In this paper, the emphasis will be more along the lines of [24]-[26].

\section{B. Notations and Abbreviations}

- $W_{L}=e^{-j 2 \pi / L}$ with the subscript deleted if obvious.

- The integer $k$ is reserved for the frequency index in the DFT expressions. Throughout the paper, $k$ is therefore analogous to frequency.

- In the figures $W_{L}^{k}$ denotes one unit of cyclic delay, that is, $y(n)=x(n-1)$ with $n-1$ interpreted modulo $L$. This is analogous to $z^{-1}$ or $e^{-j \omega}$ in standard DSP block diagrams.

- The abbreviation siso stands for single-input single-output and mimo for multi-input multi-output.

- The abbreviation PU stands for paraunitary.

- Matrices and vectors are denoted by bold letters. The notations $A^{T}, A^{*}$, and $A^{\dagger}$ denote, respectively, the transpose, the conjugate, and the transpose-conjugate of $\boldsymbol{A}$.

- The tilde notation is defined as follows: $\tilde{\boldsymbol{H}}(z)=$ $\boldsymbol{H}^{\dagger}\left(1 / z^{*}\right)$.

\section{Outline}

Section II introduces the basic building blocks for cyclic DSP. This includes filtering structures, cyclic difference equations, decimators, expanders, polyphase representations of cyclic filter banks, Nyquist property, and so forth. Section III considers cyclic versions of allpass and paraunitary properties and introduces cyclic orthonormal filter banks. Section IV studies some basic differences between the cyclic and noncyclic cases. For example, we show that a cyclic-LTI system can be paraunitary or allpass, even though the noncyclic counterpart (defined therein) may not have this property. This shows that such properties are less restrictive in the cyclic case. In Section V, we consider the paraunitary interpolation problem. We show again that noncyclic interpolants (more general than noncyclic counterparts) do not in general share the property of the underlying cyclic system. For example, we will show that there are cyclic paraunitary matrices that do not have FIR paraunitary interpolants, although IIR paraunitary interpolants always exist. In Section VI, we introduce statespace descriptions of cyclic LTI systems. We also revisit the traditional notions of reachability and observability in the context of state-space descriptions. We show that unlike in noncyclic systems, these concepts do not have a simple relation to the so-called minimality of the structure. Throughout the paper, we will point out a number of open problems pertaining to cyclic DSP systems.

\section{Basics of CyClic Digital Filters AND MulTiRATE SySTEMS}

\section{A. Filtering Structures for Cyclic Digital Filters}

Using the idea that $W_{L}^{k}$ represents a cyclic $(L)$ delay (analogous to $z^{-1}$ ), we can draw structures for cyclic-LTI systems, as demonstrated in Fig. 4. In general, this requires $L$ multipliers. By expressing the frequency response in rational form, we can sometimes obtain more efficient implementations. Thus, consider the example of a cyclic- $L$ transfer function

$$
H(k)=\frac{a_{0}+a_{1} W_{L}^{k}}{1-b W_{L}^{k}} .
$$




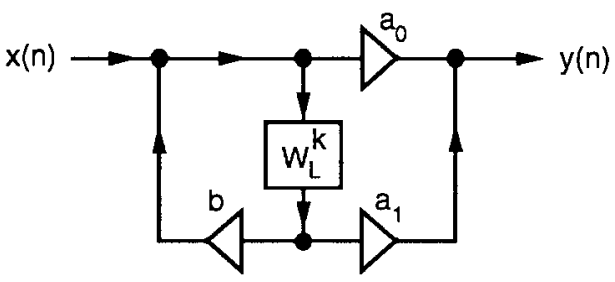

Fig. 5. Cyclic direct-form structure for a first-order filter.

The direct-form structure for this is shown in Fig. 5, and the input and output of this system are constrained by $(1-$ $\left.b W_{L}^{k}\right) Y(k)=\left(a_{0}+a_{1} W_{L}^{k}\right) X(k)$. By taking the inverse DFT of this equation, we obtain the recursive difference equation (d.e.)

$$
y(n)=b y(n-1)+a_{0} x(n)+a_{1} x(n-1)
$$

Since the time indices are interpreted modulo $L$, this is a cyclic difference equation. It is, therefore, tricky to establish an initial condition for this equation. To demonstrate why this is the case, let $L=3$. Repeated use of the difference equation [and using the facts that $y(2)=y(-1), y(3)=y(0)$ and so forth] yields the conclusion

$$
\left(1-b^{3}\right) y(0)=\alpha_{0} x(0)+\alpha_{1} x(1)+\alpha_{2} x(2)
$$

for some constants $\alpha_{i}$. Thus, the initial condition $y(0)$ is not arbitrary. It is uniquely determined as long as $b^{3} \neq 1$, that is, as long as $b \neq W_{3}^{i}$ for any $i$. This condition on $b$ is equivalent to the obvious requirement that the denominator in (2) does not become zero for any $k$. We can then write $Y(k)=H(k) X(k)$, and the inverse DFT $y(n)$ is fully determined for all $n$. More generally, consider the cyclic $(L)$ transfer function

$$
H(k)=\frac{\sum_{n=0}^{N} a_{n} W_{L}^{k n}}{1+\sum_{n=1}^{N} b_{n} W_{L}^{k n}} .
$$

With the implicit assumption that $H(k)$ is defined for all $k$ (i.e., the preceding denominator does not vanish for any $k$ ), the output is fully determined by the input. In particular, the "initial" condition is predetermined rather than arbitrary. Fig. 6 shows the direct-form implementation of this system. As a generalization of the difference equation idea, we will study in Section VI state space descriptions of cyclic LTI systems.

Even though the "initial" condition has to be computed separately, the use of a recursive structure often results in reduced computation. For example, consider the cyclic LTI system with frequency response $H(k)=\sum_{n=0}^{L-1} a^{n} W_{L}^{k n}$. This can be implemented as shown in Fig. 4, requiring $L$ multipliers and $L-1$ adders. However, we can find a more efficient recursive implementation by rewriting

$$
H(k)=\sum_{n=0}^{L-1} a^{n} W_{L}^{k n}=\frac{1-a^{L}}{1-a W_{L}^{k}} .
$$

The expression on the right-hand side yields a recursive implementation that requires only two multipliers and one adder.

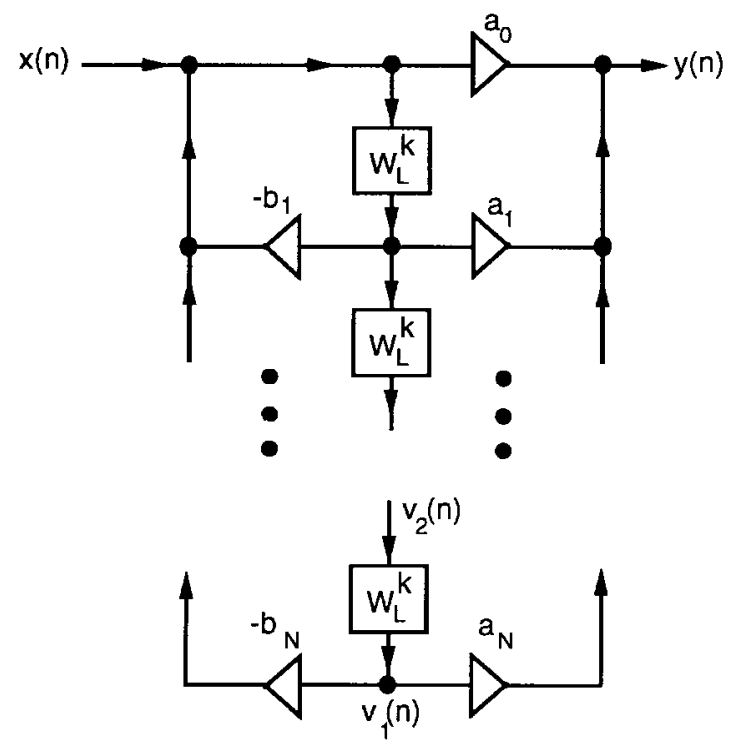

Fig. 6. Cyclic direct-form structure for a Nth-order filter.

\section{B. Cyclic Decimators and Expanders}

The cyclic decimator, denoted by $\downarrow M$ in Fig. 7(a) has the input-output relation $y(n)=x(M n)$. We assume throughout the paper that $M$ is a factor of $L$, that is ${ }^{1}$

$$
L=M K, \quad K=\text { integer. }
$$

With $x(n)$ regarded as cyclic $(L)$, the output $y(n)$ is cyclic $(K)$, as demonstrated in Fig. 7(b) and (c). Let $X(k)$ denote the $L$-point DFT of $x(n)$ and $Y(k)$ the $K$-point DFT of $y(n)$, that is, $X(k)=\sum_{n=0}^{L-1} x(n) W_{L}^{n k}, 0 \leq k \leq L-1$, and $Y(k)=\sum_{n=0}^{K-1} y(n) W_{K}^{n k}, 0 \leq k \leq K-1$. It can then be verified (Appendix A) that

$$
Y(k)=\frac{1}{M} \sum_{i=0}^{M-1} X(k-i K) \quad \text { (cyclic decimator) }
$$

for $0 \leq k \leq K-1$. Thus, the DFT $Y(k)$ is obtained by superposing $M$ shifted copies of the DFT $X(k)$, where the shifts are in multiplies of $K$. This is the counterpart of the traditional aliasing formula for decimators [23]. The cyclic expander, which is denoted by $\uparrow M$ in Fig. 8(a), has a periodic- $K$ input $x(n)$ and periodic- $L$ output $y(n)$ related by

$$
y(n)= \begin{cases}x(n / M), & n=\text { mul. of } M \\ 0, & \text { otherwise. }\end{cases}
$$

This is demonstrated in Fig. 8(b) and (c). The corresponding DFT relation is

$$
Y(k)=X(k) \quad \text { (cyclic expander) }
$$

for $0 \leq k \leq L-1$. (This is similar to the periodic extension idea in DFT theory [8]). Since $X(k)$ are the $K$-point DFT coefficients of $x(n)$, the $L$-point DFT $Y(k)$ has the period $K=L / M$. We can define fractional decimators in a manner

\footnotetext{
${ }^{1}$ If this is not the case, then decimation by $M$ would retain more samples than one-out-of $M$. For example, if $M$ and $L$ have no common factors, then there is no loss of samples at all, and the decimated output is a permuted version of the input samples.
} 


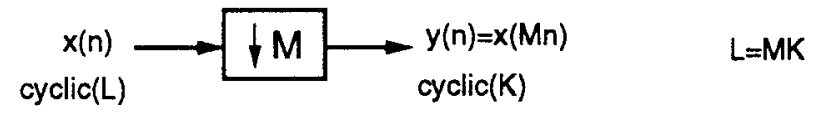

(a)

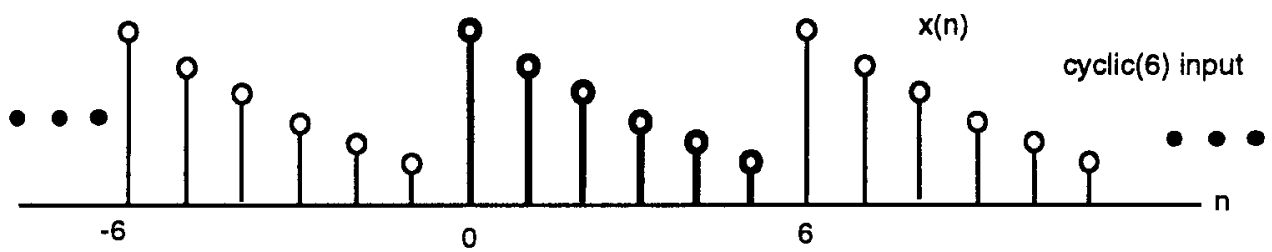

(b)

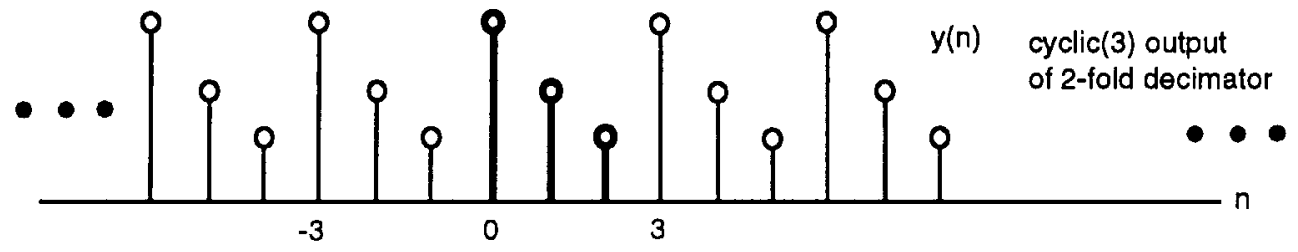

(c)

Fig. 7. (a) $M$-fold decimation of $\operatorname{cyclic}(L)$ input. (b) Example of a cyclic(6) input. (c) Two-fold decimated version.

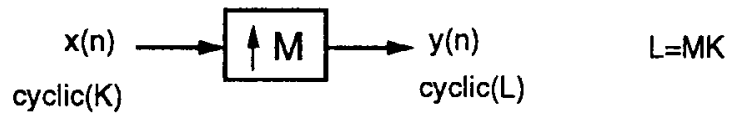

(a)

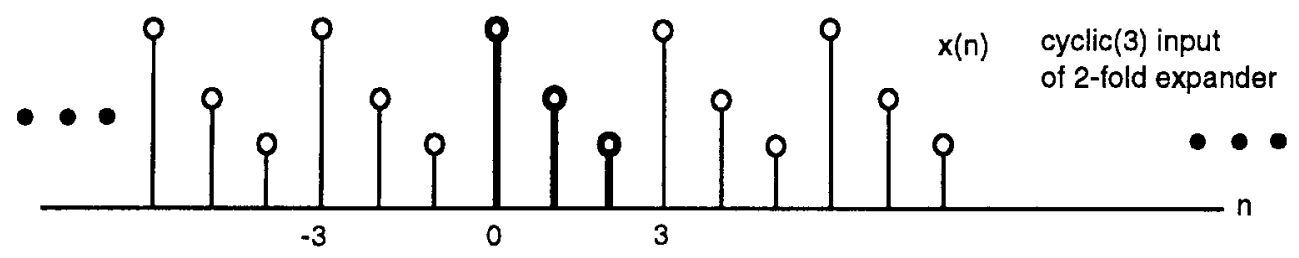

(b)

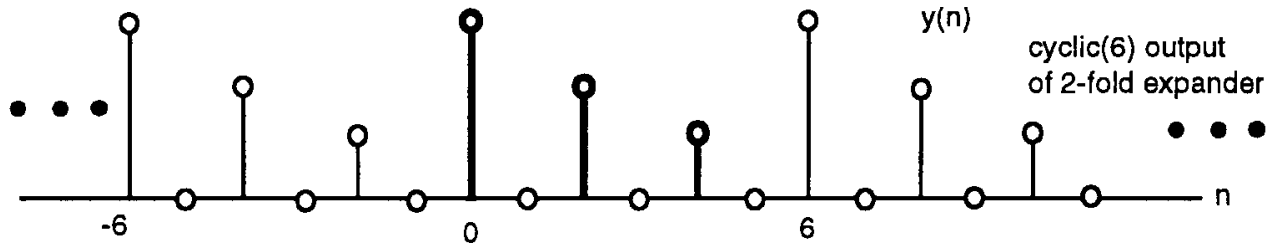

(c)

Fig. 8. (a) $M$-fold expander for $\operatorname{cyclic}(K)$ input. (b) Example of a cyclic(3) input. (c) Two-fold expanded version.

analogous to the noncyclic case (for example, as in [23, Figs. 4.1-10]). We leave it to the reader to figure out the details.

\section{Polyphase Representation}

The $L$-point DFT of a cyclic(L) impulse response $h(n)$ can be expressed as

$$
\begin{aligned}
H(k) & =\sum_{n=0}^{L-1} h(n) W_{L}^{n k}=\sum_{m=0}^{M-1} W_{L}^{k m} \sum_{n=0}^{K-1} h(M n+m) W_{K}^{k n} \\
& =\sum_{m=0}^{M-1} W_{L}^{k m} E_{m}(k)
\end{aligned}
$$

for $0 \leq k \leq L-1$, where

$$
E_{m}(k)=\sum_{n=0}^{K-1} h(M n+m) W_{K}^{k n}, \quad 0 \leq k \leq L-1 .
$$

Thus, $E_{m}(k)$ is the $K$-point DFT of the $m$ th polyphase component

$$
e_{m}(n) \triangleq h(M n+m)
$$

From the definition of $E_{m}(k)$ we see that it is $\operatorname{cyclic}(K)$. Equation (10) is analogous to the traditional Type 1 polyphase decomposition $H(z)=\sum_{k=0}^{M-1} z^{-k} E_{k}\left(z^{M}\right)$. Similarly, the Type 2 polyphase form is given by $H(k)=$ $\sum_{m=0}^{M-1} W_{L}^{-k m} R_{m}(k)$. Thus, the decimation filter of Fig. 9(a) 


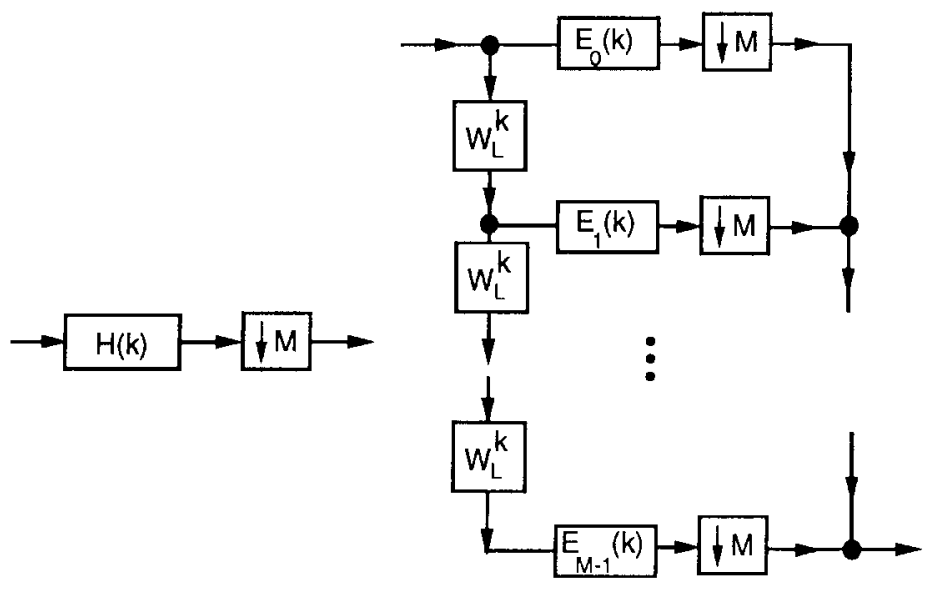

(a) (b)

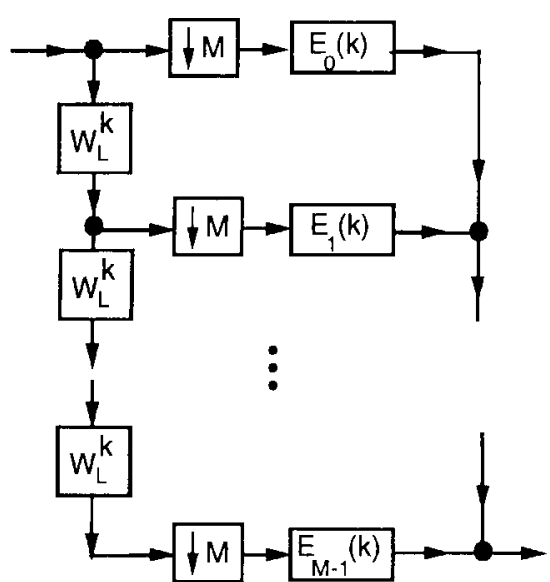

(c)

Fig. 9. (a) Decimation filter. (b) Cyclic-polyphase form. (c) Simplification using cyclic noble identity.

can be redrawn in polyphase form, as shown in Fig. 9(b). The form in Fig. 9(c) is obtained by using noble identities. This is explained next, including the reason for using the same notation $E_{m}(k)$ before and after the decimator.

Noble Identities: Since the polyphase components have a smaller period $K$, they can be relocated to the right of the decimators (similar to the use of noble identities in traditional noncyclic case). Thus, consider Fig. 10(a), where we have denoted the outputs of $E_{m}(k)$ and the decimator by $u(n)$ and $v(n)$, respectively. Let $U(k)$ be the $L$-point DFT of $u(n)$ and $V(k)$ the $K$-point DFT of $v(n)$. Then

$$
\begin{aligned}
V(k) & =\frac{1}{M} \sum_{i=0}^{M-1} U(k+i K) \\
& =\frac{1}{M} \sum_{i=0}^{M-1} X(k+i K) E_{m}(k+i K) \\
& =\frac{E_{m}(k)}{M} \sum_{i=0}^{M-1} X(k+i K)
\end{aligned}
$$

where the last line follows because we have $E_{m}(k)=$ $E_{m}(k+i K)$. However, the last line is merely the output of the system shown in Fig. 10(b), which therefore establishes the noble identity. Fig. 9(c) shows the polyphase implementation redrawn using this result. The noble identity for the expander is similar.

A caution regarding notation, however, is in order. We have used the same notation $E_{m}(k)$ for the $m$ th polyphase component in Fig. 9(b) and (c). This is regarded as a $K$ point DFT in Fig. 9(c) and an $L$-point DFT (with values repeating after a shorter period $K$ ) in Fig. 9(b). The (cyclic) impulse response of the filter $E_{m}(k)$ is accordingly as shown in Fig. 10, before and after the decimator. If this distinction is not clear from the context, we have to use a superscript as in $E_{m}^{(L)}(k)$ (before decimator) and $E_{m}^{(K)}(k)$ (after decimator).

Now, consider the analysis/synthesis system of Fig. 11(a). With the filters $H_{i}(k)$ represented in Type 1 polyphase form and the filters $F_{i}(k)$ in Type 2 form, we have the equivalent representation of Fig. 11(b), where $\boldsymbol{E}(k)$ and $\boldsymbol{R}(k)$ are the polyphase matrices of the cyclic filter bank. These should be

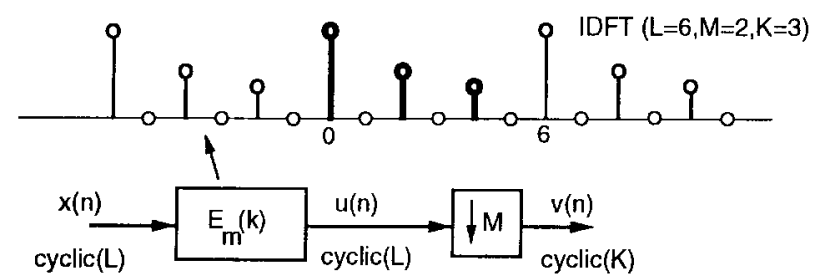

(a)

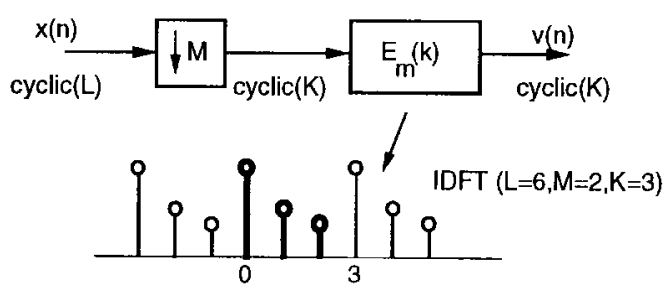

(b)

Fig. 10. Noble identities for cyclic multirate systems. (a) Polyphase component before decimator. (b) Polyphase component after decimator.

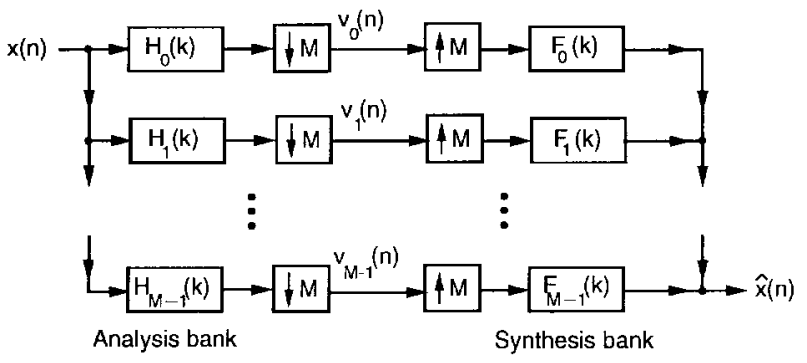

(a)

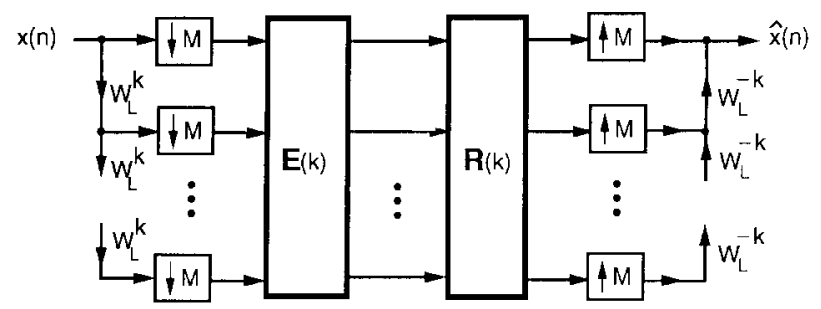

(b)

Fig. 11. (a) $\operatorname{Cyclic}(L)$ filter bank. (b) Its polyphase form. 
interpreted as $K$-point DFT's, e.g.,

$$
\boldsymbol{E}(k)=\sum_{n=0}^{K-1} \boldsymbol{e}(n) W_{K}^{k n} .
$$

The filter bank has the perfect reconstruction property $\hat{x}(n)=x(n)$ if and only if the equation $\boldsymbol{R}(k) \boldsymbol{E}(k)=\boldsymbol{I}$ is satisfied for all the $K$ values of $k$. With a slightly more general definition, we can obtain the analog of $[23,(5.6 .7)]$. The aliascomponent (AC) matrix, which is very useful in the noncyclic case [23], can also be defined for the cyclic case, and the aliasfree and perfect reconstruction conditions can be formulated using this. See Appendix B, which also shows the relation between the cyclic-AC matrix $\boldsymbol{H}(k)$ and the polyphase matrix $\boldsymbol{E}(k)$, which can be used to express orthonormality directly in terms of the filters $h_{i}(n)$.

\section{Allpass AND Paraunitary Properties}

The allpass property and, more generally, the paraunitary property play a crucial role in digital filtering and in the theory and implementation of multirate filter banks. We now extend these ideas to the cyclic case.

\section{A. Cyclic Allpass Filters}

A cyclic $(L)$ allpass system is one for which $H(k)=e^{j \phi(k)}$ for $0 \leq k \leq L-1$. A simple example is the first-order system

$$
H(k)=\frac{b^{*}+W_{L}^{k}}{1+b W_{L}^{k}} .
$$

We can always rewrite any cyclic allpass system $H(k)=$ $e^{j \phi(k)}$ in rational form

$$
H(k)=\frac{c \sum_{n=0}^{J} b_{J-n}^{*} W_{L}^{k n}}{\sum_{n=0}^{J} b_{n} W_{L}^{k n}}, \quad|c|=1 .
$$

For example, we can let $J=L-1$ and set $e^{-j \phi(k) / 2}=$ $\sum_{n=0}^{L-1} b_{n} W_{L}^{k n}$. The coefficients $b_{n}$ are essentially the inverse DFT coefficients of $e^{-j \phi(k) / 2}$ and can readily be identified. More interesting is the open problem of obtaining the rational form with smallest order $J$.

The allpass property in the cyclic case can be expressed entirely in terms of the unitary property of a circulant matrix. For this, consider the $L \times L$ circulant matrix $\boldsymbol{H}$ formed from the impulse response $h(k)$ demonstrated below for $L=4$.

$$
\boldsymbol{H}=\left[\begin{array}{llll}
h(0) & h(1) & h(2) & h(3) \\
h(3) & h(0) & h(1) & h(2) \\
h(2) & h(3) & h(0) & h(1) \\
h(1) & h(2) & h(3) & h(0)
\end{array}\right]
$$

We know that circulant matrices are diagonalized by the DFT matrix (which is unitary) and that the eigenvalues are the DFT coefficients $H(k)$. That is,

$$
H=\frac{W \Lambda W^{\dagger}}{M}
$$

where $W^{\dagger} W=M \boldsymbol{I}$, and $\boldsymbol{A}$ is a diagonal matrix with elements $H(k)$. Using this, we can see that $\boldsymbol{H}^{\dagger} \boldsymbol{H}=\boldsymbol{I}$ if and only if $|H(k)|=1$. Thus, the cyclic allpass property is equivalent to the unitariness of the matrix $\boldsymbol{H}$.

The rational form (15) has nonzero denominator for all $k$ as long as the $L$-point DFT of $\left\{b_{n}\right\}$ is nonzero for all $k$. Equivalently, the polynomial $\Sigma_{n} b_{n} z^{-n}$ should have no zeros at the unit-circle points $z=W_{L}^{k}$. For the allpass case, we can assume further that $\Sigma_{n} b_{n} z^{-n}$ has no zeros anywhere on the unit circle because such a zero would also be present in $\sum_{n=0}^{J} b_{J-n}^{*} z^{-n}$ and can be cancelled anyway. Thus, any cyclic allpass system can be written as in (15), where $b_{n}$ are such that $\Sigma_{n} b_{n} z^{-n}$ is free from unit circle zeros.

\section{B. Cyclic Paraunitary Systems and Orthonormal Filter Banks}

The $M \times M$ cyclic transfer matrix $\boldsymbol{E}(k)$ is said to be cyclic-paraunitary (or cyclic-PU) if it is unitary for all $k$. In Sections IV and V, we consider the properties of cyclicPU systems in greater detail and show that they do not share some of the restrictions of noncyclic PU systems.

We define the $M$-band cyclic filter bank [Fig. 11(a)] to be orthonormal if the polyphase matrix $\boldsymbol{E}(k)$ is unitary for all $k$. That is, $\boldsymbol{E}(k)$ is cyclic-PU. The perfect reconstruction property then reduces to $\boldsymbol{R}(k)=\boldsymbol{E}^{\dagger}(k)$, which can be rewritten in terms of the $\operatorname{cyclic}(L)$ impulse responses as

$$
f_{i}(n)=h_{i}^{*}(-n) .
$$

The DFT's are correspondingly related as $F_{i}(k)=H_{i}^{*}(k)$. Recall here that the arguments of $h_{i}(\cdot), f_{i}(\cdot), H_{i}(\cdot)$, and $F_{i}(\cdot)$ are interpreted modulo $L$. The above definition of orthonormality is consistent with the statement that the filter bank expands the cyclic $(L)$ signal $x(n)$ using an orthonormal basis. Thus, assuming $\hat{x}(n)=x(n)$ in Fig. 11(a), we have

$$
x(n)=\sum_{i=0}^{M-1} \sum_{\ell=0}^{K-1} v_{i}(\ell) f_{i}(n-\ell M), \quad 0 \leq n \leq L-1 .
$$

The basis functions are the length- $L$ sequences

$$
\eta_{i, \ell}(n) \triangleq f_{i}(n-\ell M), \quad 0 \leq n \leq L-1
$$

where $0 \leq i \leq M-1,0 \leq \ell \leq K-1$. Thus, there are $M K=L$ basis functions $\eta_{i, \ell}(n)$. It can be shown that the unitarity of $\boldsymbol{R}(k)$ is equivalent to orthonormality of the basis $\eta_{i, \ell}(n)$ (Appendix B). This orthonormality can be reexpressed as

$$
\sum_{n=0}^{L-1} f_{i}(n) f_{m}^{*}(n-M \ell)=\delta(i-m) \delta(\ell)
$$

As in traditional filter banks, orthonormality of the cyclic filter bank implies the following:

1) Unit-energy property: $\Sigma_{n=0}^{L-1}\left|f_{i}(n)\right|^{2}=1$ for all $i$ [and, similarly, for $\left.h_{i}(n)\right]$.

2) Power complementary property: $\sum_{i=0}^{M-1}\left|F_{i}(k)\right|^{2}=M$, for all $k$ [and, similarly, for $\left.H_{i}(k)\right]$. 


\section{Cyclic Versus Noncyclic Systems}

There are several basic differences between the behaviors of cyclic and noncyclic LTI systems. To demonstrate, consider the determinant of a paraunitary matrix. For the noncyclic case, this can be shown to be an allpass function [23]. If $\boldsymbol{E}(k)$ is cyclic paraunitary, then the same result can be proved, that is, $[\operatorname{det} \boldsymbol{E}(k)]=e^{j \phi(k)}=$ allpass. However, a difference in behavior arises when we try to relate the degree of determinant to the degree of the system. The degree (or McMillan degree) of a noncyclic system $\boldsymbol{E}_{\text {non }}(z)$ is defined as the minimum number of delay elements $z^{-1}$ required to implement it. By analogy, in the cyclic case, suppose we define the degree of $\boldsymbol{E}(k)$ to be the minimum number of cyclic delay elements $W_{L}^{k}$ required to implement $\boldsymbol{E}(k){ }^{2}$ For noncyclic FIR paraunitary systems, the degree of $\left[\operatorname{det} \boldsymbol{E}_{\mathrm{non}}(z)\right]$ is equal to the degree of $\boldsymbol{E}_{\text {non }}(z)$ (see [23]), but the same is not true in the cyclic case. For example, consider the cyclic paraunitary system

$$
\boldsymbol{E}(k)=\left[\begin{array}{cc}
\cos \theta(k) & \sin \theta(k) \\
-\sin \theta(k) & \cos \theta(k)
\end{array}\right], \quad 0 \leq k \leq L-1 .
$$

Here, $[\operatorname{det} \boldsymbol{E}(k)]=1$. Thus, regardless of the degree of $\boldsymbol{E}(k)$, the determinant has degree equal to zero.

Another difference pertains to factorizability. It is wellknown that noncyclic FIR paraunitary systems can be factored [23] in terms of degree-one FIR building blocks. However, in the cyclic case, such factorization is not always possible, as explained at the end of Section V-D.

\section{A. The Noncyclic Counterpart}

In the cyclic $(L)$ case, any transfer function can be expressed in the form $H(k)=\Sigma_{n=0}^{L-1} h(n) W_{L}^{k n}$. The noncyclic counterpart of this is defined as

$$
H_{n c}(z)=\sum_{n=0}^{L-1} h(n) z^{-n} .
$$

This can be regarded as an interpolated version in the frequency domain, with $H(k)$ representing the samples of $H_{n c}(z)$ at the unit-circle points $z=W_{L}^{-k}=e^{j 2 \pi k / L}$. Similarly, the noncyclic counterpart of the $M \times M$ cyclic $(L)$ system $\boldsymbol{E}(k)=\Sigma_{n=0}^{L-1} \boldsymbol{e}(n) W_{L}^{k n}$ is defined by $\boldsymbol{E}_{n c}(z)=$ $\sum_{n=0}^{L-1} e(n) z^{-n}$.

The interpolated version or interpolant, however, is not unique. For example, we can find a noncyclic interpolant $G_{\text {int }}(z)=\sum_{n=0}^{N} g(n) z^{-n}$ with $N>L-1$ such that $G_{\text {int }}\left(W_{L}^{-k}\right)=H(k)$. As another example, consider the cyclic system

$$
\sum_{n=0}^{L-1} a^{n} W_{L}^{k n}=\frac{1-a^{L}}{1-a W_{L}^{k}} .
$$

If we replace $W_{L}^{k}$ with $z^{-1}$ to obtain an interpolant, then the answer obtained from the left-hand side is different from what

\footnotetext{
${ }^{2}$ Notice that the cyclic nature of time makes this rather unnatural. For example, the system $H(k)=W_{L}^{(L-1) k}$ requires $L-1$ cyclic delays but can be rewritten as $H(k)=W_{L}^{-k}$, requiring only one cyclic advance operator $W_{L}^{-k}$.
}

we get from the right-hand side. Two possible interpolants in this case are, therefore, $\sum_{n=0}^{L-1} a^{n} z^{-n}$ and $\left(1-a^{L}\right) /(1-a z)^{-1}$. It is clear that the noncyclic counterpart is only one of the many possible interpolants. ${ }^{3}$

If $\boldsymbol{E}_{n c}(z)$ is PU, it readily follows that $\boldsymbol{E}(k)$ is cyclic-PU because each $k$ corresponds to a special $z$ on the unit circle. However, the converse does not hold, as we shall see. Thus, cyclic paraunitariness is less of a constraint on the coefficients $\boldsymbol{e}(n)$ than traditional paraunitariness. To demonstrate, consider the second-order cyclic(4) transfer function

$$
G(k)=0.5+0.5(j-1) W_{4}^{k}+0.5 j W_{4}^{2 k}
$$

Using the facts that $W_{4}^{4}=1$ and $W_{4}^{2}=-1$, it is readily verified that $|G(k)|^{2}=1$ for all $k$. Thus, $G(k)$ is allpass in the cyclic(4) sense. However, the noncyclic counterpart $G_{n c}(z)=0.5+0.5(j-1) z^{-1}+0.5 j z^{-2}$, which is an FIR filter, is evidently not allpass. If we now construct the $M \times M$ polyphase matrix $\boldsymbol{E}(k)=G(k) \boldsymbol{I}_{M}$ (for arbitrary $M$ ) then it is cyclic paraunitary, although the noncyclic counterpart $\boldsymbol{E}_{n c}(z)=G_{n c}(z) \boldsymbol{I}$ is not paraunitary.

As a second example, consider the cyclic(3) analysis bank

$$
\boldsymbol{H}(k)=\left[\begin{array}{c}
H_{0}(k) \\
H_{1}(k) \\
H_{2}(k)
\end{array}\right]=\boldsymbol{a}_{0}+\boldsymbol{a}_{1} W_{3}^{k}+\boldsymbol{a}_{2} W_{3}^{2 k}
$$

where $a_{i}$ are the column-vectors given by

$$
\boldsymbol{a}_{0}=\frac{1}{\sqrt{10}}\left[\begin{array}{l}
1 \\
1 \\
0
\end{array}\right], \quad \boldsymbol{a}_{1}=\frac{1}{\sqrt{10}}\left[\begin{array}{c}
1 \\
-1 \\
-2
\end{array}\right], \quad \boldsymbol{a}_{2}=\frac{1}{\sqrt{10}}\left[\begin{array}{l}
1 \\
0 \\
1
\end{array}\right] .
$$

It can be verified that $\boldsymbol{H}^{\dagger}(k) \boldsymbol{H}(k)=1$, that is, the three transfer functions $H_{0}(k), H_{1}(k)$ and $H_{2}(k)$ satisfy the cyclic power complementary property

$$
\left|H_{0}(k)\right|^{2}+\left|H_{1}(k)\right|^{2}+\left|H_{2}(k)\right|^{2}=1, \quad k=0,1,2 .
$$

Consider the noncyclic counterpart $\boldsymbol{H}_{n c}(z)=\boldsymbol{a}_{0}+\boldsymbol{a}_{1} z^{-1}+$ $\boldsymbol{a}_{2} z^{-2}$. By explicit calculation, we find that $\tilde{\boldsymbol{H}}_{n c}(z) \boldsymbol{H}_{n c}(z) \neq$ 1 (e.g., the coefficient of $z^{-2}$, which is $\boldsymbol{a}_{0}^{\dagger} \boldsymbol{a}_{2}$, is nonzero). Thus, the noncyclic counterpart is not power complementary, although the cyclic(3) system is.

\section{B. Nyquist Property, Linear-Phase, and CQF Design}

The next example brings out another difference between cyclic and noncyclic filters. Consider a cyclic(6) transfer function

$$
H_{0}(k)=\left(1+W_{6}^{k}-W_{6}^{2 k}+W_{6}^{3 k}+W_{6}^{4 k}\right) / \sqrt{5}
$$

whose impulse response is shown in Fig. 12(a). We can regard this as FIR in the sense that $h(n)$ is nonzero only on a subset of points in $0 \leq n \leq L-1$. (In the cyclic case, this is the only FIR definition that makes sense). The symmetry of the impulse response implies the linear-phase property, as explicitly seen from

$$
H_{0}(k)=W_{6}^{2 k}(-1+2 \cos (\pi k / 3)+2 \cos (2 \pi k / 3) / \sqrt{5} .
$$

${ }^{3}$ The subscript $n c$ will be for "noncyclic counterpart." We will use the subscript int to refer to any interpolated version and the subscript non to indicate any noncyclic system. 


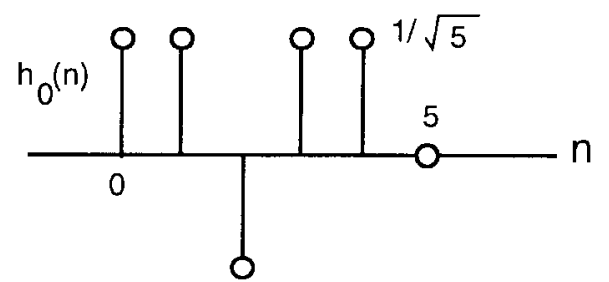

(a)

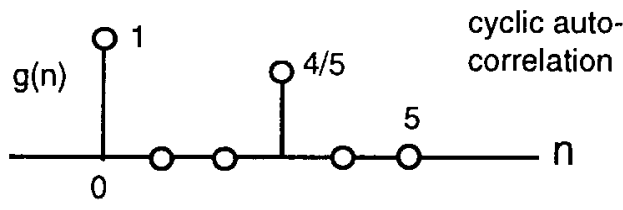

(b)

Fig. 12. (a) Cyclic(6) linear-phase FIR filter. (b) Its autocorrelation, which is a halfband filter.

Now, consider the magnitude-squared function $G(k)=$ $\left|H_{0}(k)\right|^{2}$, whose cyclic(6) impulse response $g(n)$ is the cyclic convolution of $h_{0}(n)$ with $h_{0}^{*}(-n)$. By explicit computation, we verify that $g(0)=1$, and $g(2)=g(4)=0$ [Fig. 12(b)]. That is, $g(2 n)=\delta(n)$. Therefore, the filter $G(k)$ has the halfband property [in the cyclic(6) sense], and

$$
G(k)+G(3+k)=2, \quad 0 \leq k \leq 5 .
$$

Equivalently, $H(k)$ is power symmetric [23] in the cyclic(6) sense

$$
\left|H_{0}(k)\right|^{2}+\left|H_{0}(3+k)\right|^{2}=2, \quad 0 \leq k \leq 5 .
$$

That is, we have found a cyclic(6) filter $H_{0}(k)$ that is both linear phase and power symmetric. This is not possible [23] for the noncyclic FIR case.

Using the above example, we can construct a two-channel cyclic(6) orthonormal filter bank where the filters are nontrivial linear-phase filters. (Such constructions are not possible in the noncyclic FIR case [23].) For this, choose $h_{0}(n)$ as above and the remaining three filters as

$$
h_{1}(n)=(-1)^{n} h_{0}^{*}(1-n), \quad f_{k}(n)=h_{k}^{*}(-n)
$$

for $k=0,1$, with all arguments interpreted modulo- 6 . That is, $H_{1}(k)=-W_{L}^{k} H_{0}^{*}(k-K)$, where $K=L / 2$, and $F_{i}(k)=$ $H_{i}^{*}(k)$. The preceding is an example of a cyclic(6) version of the CQF design of Smith and Barnwell [18].

More on the CQF Design: Given a cyclic $(L)$ impulse response $g(n)$ with the property that $G(k) \geq 0$, we can construct infinitely many cyclic $(L)$ two-channel orthonormal filter banks by choosing

$$
H_{0}(k)=e^{j \phi(k)} \sqrt{G(k)}
$$

and the remaining three filters according to (25). We can regard $H_{0}(k)$ as a cyclic spectral factor of $G(k)$. Recall that for noncyclic FIR filters, the usual definition of spectral factors allows only finitely many phase responses, and the spectral factors all have the same length $N+1$. In (26), however, the phase response $\phi(k)$ of the spectral factor $H_{0}(k)$ is arbitrary. In particular, the choice $\phi(k)=0$ would

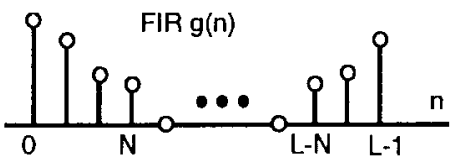

(a)

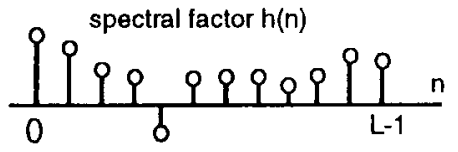

(b)

Fig. 13. (a) Cyclic( $L$ ) FIR filter. (b) Its non-FIR spectral factor.

yield linear-phase analysis filters $H_{0}(k)$ and $H_{1}(k)$ with good frequency responses [if $G(k)$ is a good lowpass filter]. However, even if $G(k)$ is $\operatorname{cyclic}(L)$ FIR [in the sense that $g(n)=0$ for $N<n<L-N$; see Fig. 13), the cyclic spectral factor $H_{0}(k)$ may not be FIR [i.e., $h_{0}(k)$ could be nonzero for all $n]$. An open question here is what is the most general form of the phase response $\phi(k)$ that ensures that $H_{0}(k)$ is FIR (possibly with length $N+1$ )? A related question is the following: For a given $G(k)$, what is the minimum-length linear-phase spectral factor?

\section{The PU InTERPolation PROBLEM}

Given an arbitrary $\operatorname{cyclic}(L)$ paraunitary system $\boldsymbol{H}(k)$, that is, a sequence of $M \times M$ unitary matrices

$$
\boldsymbol{H}(0), \boldsymbol{H}(1), \cdots, \boldsymbol{H}(L-1)
$$

can we always find a paraunitary interpolant? That is, can we find a system $\boldsymbol{H}_{\text {int }}\left(e^{j \omega}\right)=\sum_{n} h(n) e^{-j \omega n}$ such that its samples at the unit circle points $z=W_{L}^{-k}$ agree with $\boldsymbol{H}(k)$ ? This is the paraunitary interpolation problem. For the scalar case $(M=1)$, this becomes the allpass interpolation problem. For the matrix case (arbitrary $M$ ), we distinguish between the FIR interpolation problem, where $\boldsymbol{H}_{\text {int }}(z)=\sum_{n=0}^{N} \boldsymbol{h}(n) z^{-n}$, and the IIR case. In the IIR case, we again distinguish between rational interpolants [where each element in $\boldsymbol{H}_{\text {int }}(z)$ is a ratio of polynomials in $z^{-1}$ ] and irrational ones. Notice that if the order of the interpolant $N<L$, then $\boldsymbol{H}_{\text {int }}(z)$ is the unique noncyclic counterpart $\boldsymbol{H}_{n c}(z)$ defined in Section IV-A, which is, in general, not PU. Things become more interesting when $N \geq L$.

\section{A. Scalar Allpass Interpolation}

Any scalar cyclic allpass filter $H(k)$ can be written in the form (15), which shows that there exists a noncyclic allpass interpolant $H_{\text {int }}(z)=\left(c \sum_{n=0}^{J} b_{J-n}^{*} z^{-n}\right) /\left(\sum_{n=0}^{J} b_{n} z^{-n}\right)$, where $|c|=1$. This interpolant is IIR, and, in general, does not have all poles inside the unit circle. For example, suppose $N=1$, and consider the cyclic allpass filter $H(k)$ and its interpolant $H_{\text {int }}(z)$ given as

$$
H(k)=\frac{b^{*}+W_{L}^{k}}{1+b W_{L}^{k}} \quad H_{\mathrm{int}}(z)=\frac{b^{*}+z^{-1}}{1+b z^{-1}} .
$$

Let $|b|>1$. Then, the cyclic allpass filter is still well defined (because $1+b W_{L}^{k} \neq 0$ for any $k$ ). However, the interpolant 
$H_{\text {int }}(z)$ represents an allpass filter with a pole outside the unit circle. This raises the following question: Suppose we allow the allpass interpolant to be of higher order $N$ (even possibly $N>L)$. That is, we now have

$$
\begin{aligned}
H(k) & =\frac{b^{*}+W_{L}^{k}}{1+b W_{L}^{k}} \\
H_{\text {int }}(z) & =\frac{c \sum_{n=0}^{N} b_{N-n^{*}}^{*} z^{-n}}{\sum_{n=0}^{N} b_{n} z^{-n}}=\frac{c z^{-N} \tilde{B}_{\text {int }}(z)}{B_{\text {int }}(z)} .
\end{aligned}
$$

Can we show that there exists a polynomial $\sum_{n=0}^{N} b_{n} z^{-n}$ of some order $N$ with all its zeros inside the unit circle such that $H(k)=H_{\text {int }}\left(W_{L}^{-k}\right)$ ? While the answer to this is not clear, the following example gives evidence to the contrary.

Example 1-Minimum-Phase Interpolants: Let $B(k)=$ $1+b W_{L}^{k}$ with $b>1$, and let $B_{\text {int }}(z)=\sum_{n=0}^{N} b_{n} z^{-n}$ be an interpolant $B(k)=B_{\text {int }}\left(W_{L}^{-k}\right)$. In this example, we restrict the coefficients $b_{n}$ to also be real. We can find unlimited number of choices of $N$ and $\left\{b_{n}\right\}$ satisfying these conditions. However, for even $L$, there does not exist even one choice such that $B_{\text {int }}(z)$ has minimum phase (i.e., all zeros in $|z|<1$ ). This is proved as follows: Since the coefficients $\left\{b_{n}\right\}$ are real, a necessary condition for $B_{\text {int }}(z)$ to have minimum phase is that $B_{\text {int }}(1)$ and $B_{\text {int }}(-1)$ have the same sign [2]. By setting $k=0$ in $B(k)=B_{\text {int }}\left(W_{L}^{-k}\right)$, we find $B(0)=B_{\text {int }}(1)$. Similarly, $B(L / 2)=B_{\text {int }}(-1)$. Using $b>1$, we therefore conclude

$$
\begin{aligned}
B_{\text {int }}(1) & =B(0)=1+b>0 \\
B_{\text {int }}(-1) & =B(L / 2)=1-b<0
\end{aligned} .
$$

Thus, $B_{\text {int }}(1)$ and $B_{\text {int }}(-1)$ could never have the same sign no matter how we choose $N$ and $\left\{b_{n}\right\}$. Summarizing, $B(k)$ does not have a minimum-phase interpolant $B_{\text {int }}(z)$. However, it is still conceivable that the ratio $\tilde{B}_{\text {int }}(z) / B_{\text {int }}(z)$ has some cancellations, thereby resulting in a stable allpass interpolant. Moreover, we have not considered the possibility of a complex-coefficient interpolant (which is conceivable even for real $b$ ).

\section{B. FIR PU Interpolation}

Given the unitary sequence (27), can we always find a $M \times M$ PU interpolant restricted to be FIR, i.e., of the form $\boldsymbol{H}_{\text {int }}(z)=\sum_{n=0}^{N} \boldsymbol{h}(n) z^{-n}$ ? For the scalar case $(M=1)$, the answer is evidently no because the interpolant has to be an FIR allpass function (which cannot be more general than a mere delay). Therefore, we assume $M \neq 1$. If we make the restriction $N<L$, the coefficients $h(n)$ are simply the inverse DFT coefficients of $\boldsymbol{H}_{\text {int }}\left(W_{L}^{-k}\right)$ and the interpolant is the noncyclic counterpart defined in Section IV-A. This may not be PU as seen from the examples of Section IV-A. More generally, suppose we allow $N$ to be arbitrarily large but finite. Does this allow us to always find a paraunitary interpolant? In general, the answer is still no, as we shall demonstrate. For this, we first review a well-known result for noncyclic $2 \times 2$ causal FIR paraunitary matrices [23].
Theorem 1: Let $\boldsymbol{H}_{\text {non }}(z)=\sum_{n=0}^{N} \boldsymbol{h}(n) z^{-n}$ be a $2 \times 2$ causal FIR system. Then, it is paraunitary if and only if it has the form

$$
\boldsymbol{H}_{\text {non }}(z)=\left[\begin{array}{cc}
H_{0}(z) & e^{j \theta} z^{-n_{0}} \tilde{H}_{1}(z) \\
H_{1}(z) & -e^{j \theta} z^{-n_{0}} \tilde{H}_{0}(z)
\end{array}\right]
$$

where $\tilde{H}_{0}(z) H_{0}(z)+\tilde{H}_{1}(z) H_{1}(z)=1$ (power complementary property), $\theta$ is real, and $n_{0}$ is any integer large enough to ensure causality.

A proof is included in Appendix $\mathrm{C}$ for completeness. Consider now the $\operatorname{cyclic}(L)$ example

$$
\boldsymbol{H}(k)=\left[\begin{array}{cc}
e^{j \alpha(k)} & 0 \\
0 & e^{j \beta(k)}
\end{array}\right]
$$

which is evidently paraunitary for any arbitrary (real-valued) $\alpha(k)$ and $\beta(k)$. Suppose there exists a noncyclic causal FIR paraunitary interpolant $\boldsymbol{H}_{\text {int }}(z)$. If this interpolant is diagonal, then $H_{0}\left(e^{j \omega}\right)=c e^{-j \omega N}$ and $\alpha(k)$ has to be of the form $-2 \pi k N / L$ plus a constant. Thus, for arbitrary $\alpha(k)$ and $\beta(k)$, there is no diagonal FIR paraunitary interpolant $\boldsymbol{H}_{\text {int }}(z)$. How about a nondiagonal interpolant? Since it would have the form (30), $\alpha(k)$ and $\beta(k)$ cannot have arbitrary combinations of values. Only those combinations that satisfy the property

$$
\beta(k)=-\alpha(k)-2 \pi k n_{0} / L+\delta
$$

for some $\delta$ are allowed. This shows that cyclic PU systems do not in general have noncyclic FIR paraunitary interpolants. Whenever such an interpolant does exist, it can be factorized into the form [23]

$$
\begin{aligned}
\boldsymbol{H}_{\mathrm{int}}(z)= & \boldsymbol{H}_{\mathrm{int}}(1)\left(\boldsymbol{I}-\boldsymbol{u}_{1} \boldsymbol{u}_{1}^{\dagger}+z^{-1} \boldsymbol{u}_{1} \boldsymbol{u}_{1}^{\dagger}\right) \\
& \cdot\left(\boldsymbol{I}-\boldsymbol{u}_{2} \boldsymbol{u}_{2}^{\dagger}+z^{-1} \boldsymbol{u}_{2} \boldsymbol{u}_{2}^{\dagger}\right) \\
& \cdots\left(\boldsymbol{I}-\boldsymbol{u}_{J} \boldsymbol{u}_{J}^{\dagger}+z^{-1} \boldsymbol{u}_{J} \boldsymbol{u}_{J}^{\dagger}\right)
\end{aligned}
$$

where $\boldsymbol{u}_{i}$ are unit norm vectors. By replacing $z^{-1}$ with $W_{L}^{k}$, we obtain

$$
\begin{aligned}
\boldsymbol{H}(k)= & \boldsymbol{H}(0)\left(\boldsymbol{I}-\boldsymbol{u}_{1} \boldsymbol{u}_{1}^{\dagger}+W_{L}^{k} \boldsymbol{u}_{1} \boldsymbol{u}_{1}^{\dagger}\right)\left(\boldsymbol{I}-\boldsymbol{u}_{2} \boldsymbol{u}_{2}^{\dagger}+W_{L}^{k} \boldsymbol{u}_{2} \boldsymbol{u}_{2}^{\dagger}\right) \\
& \cdots\left(\boldsymbol{I}-\boldsymbol{u}_{J} \boldsymbol{u}_{J}^{\dagger}+W_{L}^{k} \boldsymbol{u}_{J} \boldsymbol{u}_{J}^{\dagger}\right)
\end{aligned}
$$

which is a factorization of the cyclic-PU system $\boldsymbol{H}(k)$ in terms of the building blocks $\left(\boldsymbol{I}-\boldsymbol{u}_{i} \boldsymbol{u}_{i}^{\dagger}+W_{L}^{k} \boldsymbol{u}_{i} \boldsymbol{u}_{i}^{\dagger}\right)$. Thus, whenever a noncyclic FIR paraunitary interpolant exists, the cyclic system can be factored as above. Conversely, if the cyclic system can be factored as in (33), then replacing $W_{L}^{k}$ with $z^{-1}$, we obtain a FIR paraunitary interpolant. Summarizing we have proved:

Theorem 2-FIR Interpolants: Let $\boldsymbol{H}(k)$ be a cyclic $(L)$ paraunitary system, i.e., $\boldsymbol{H}(k)$ unitary for $0 \leq k \leq L-1$. Then, it has a noncyclic causal FIR paraunitary interpolant $\boldsymbol{H}_{\text {int }}(z)$ if and only if $\boldsymbol{H}(k)$ can be factorized in the form (33), where $\boldsymbol{u}_{i}$ are unit-norm vectors.

\section{IIR Paraunitary Interpolation}

If we do not restrict the interpolant to be FIR, then we can always find a paraunitary interpolant for the unitary sequence (27). For this, we simply define

$$
\boldsymbol{H}_{\text {int }}\left(e^{j \omega}\right)=\boldsymbol{H}(k), \quad \frac{2 \pi k}{L} \leq \omega<\frac{2 \pi(k+1)}{L}
$$


for $0 \leq k \leq L-1$. Then, the sampled values $H_{\text {int }}\left(W_{L}^{-k}\right)$ are evidently equal to $\boldsymbol{H}(k)$. The interpolant $\boldsymbol{H}_{\text {int }}\left(e^{j \omega}\right)$ is a piecewise constant and has discontinuities at the frequencies $2 \pi k / L$. It is therefore not a rational function in $e^{j \omega}$, i.e., the elements in the matrix are not ratios of polynomials in $z$. The following theorem asserts that we can always construct rational solutions in the IIR case. Stability of the interpolant, however, is not asserted.

Theorem 3-IIR Interpolants: Let $\boldsymbol{H}(0), \boldsymbol{H}(1), \cdots \boldsymbol{H}(L-$ 1) be a sequence of $M \times M$ unitary matrices. Then, there exists a causal system with rational transfer matrix $\boldsymbol{H}_{\text {int }}(z)$ such that $\boldsymbol{H}_{\text {int }}\left(W_{L}^{-k}\right)=\boldsymbol{H}(k)$.

Proof: The crucial building block is the matrix

$$
\boldsymbol{U}_{k}(z) \triangleq \boldsymbol{I}-\boldsymbol{u} \boldsymbol{u}^{\dagger}+z^{-1} F_{k}(z) \boldsymbol{u} \boldsymbol{u}^{\dagger}
$$

where $F_{k}(z)$ is a rational allpass filter, and $\boldsymbol{u}$ is a unit-norm vector. We can verify that $\boldsymbol{U}_{k}(z)$ is PU, that is, $\widetilde{\boldsymbol{U}}_{k}(z) \boldsymbol{U}_{k}(z)=$ $\boldsymbol{I}$. Suppose the allpass filter $F_{k}(z)$ is chosen such that

$$
F_{k}\left(W_{L}^{-m}\right)= \begin{cases}W_{L}^{-m}, & m \neq k \\ -W_{L}^{-m}, & m=k .\end{cases}
$$

We can regard $F_{k}(z)$ as a rational allpass interpolant (Section V-A) with samples at $z=W_{L}^{-m}$ as specified above. With this choice of $F_{k}(z)$, the matrix $\boldsymbol{U}_{k}(z)$, sampled at $z=W_{L}^{-m}$, yields

$$
\boldsymbol{U}_{k}\left(W_{L}^{-m}\right)= \begin{cases}I, & \text { for all } m \neq k \\ I-2 \boldsymbol{u} \boldsymbol{u}^{\dagger}, & \text { for } m=k\end{cases}
$$

Now, any $M \times M$ unitary matrix can be expressed as a product of $M-1$ matrices of the form $\boldsymbol{I}-2 \boldsymbol{u} \boldsymbol{u}^{\dagger}$. More precisely [23], each matrix $\boldsymbol{H}(k)$ in the given unitary sequence can be expressed as

$$
\begin{aligned}
\boldsymbol{H}(k)= & \left(\boldsymbol{I}-2 \boldsymbol{u}_{1, k} \boldsymbol{u}_{1, k}^{\dagger}\right)\left(\boldsymbol{I}-2 \boldsymbol{u}_{2, k} \boldsymbol{u}_{2, k}^{\dagger}\right) \\
& \cdots\left(\boldsymbol{I}-2 \boldsymbol{u}_{M-1, k} \boldsymbol{u}_{M-1, k}^{\dagger}\right) \boldsymbol{\Lambda}(k)
\end{aligned}
$$

where $\boldsymbol{u}_{i, k}$ are unit-norm vectors, and $\boldsymbol{\Lambda}(k)$ is diagonal with $n$th diagonal element $e^{j \theta_{k, n}}$. We can find a rational allpass filter $F_{n}(z)$ such that

$$
F_{n}\left(W_{L}^{-m}\right)= \begin{cases}1, & m \neq k \\ e^{j \theta_{k, n}}, & m=k\end{cases}
$$

Then, the diagonal matrix $\boldsymbol{D}_{k}(z)$ with diagonal elements $F_{n}(z)$ has the unit-circle samples

$$
D_{k}\left(W_{L}^{-m}\right)= \begin{cases}I, & m \neq k \\ \Lambda(k), & m=k\end{cases}
$$

By multiplying matrices of the form $U_{k}(z)$ and $D_{k}(z)$, we can define a noncyclic paraunitary system $\boldsymbol{G}_{k}(z)$ such that

$$
\boldsymbol{G}_{k}\left(W_{L}^{-m}\right)= \begin{cases}I, & m \neq k \\ \boldsymbol{H}(k), & m=k\end{cases}
$$

The product $\boldsymbol{H}_{\text {int }}(z)=\boldsymbol{G}_{0}(z) \boldsymbol{G}_{1}(z) \cdots \boldsymbol{G}_{L-1}(z)$ then represents a rational IIR PU interpolant for the given matrix sequenence $\{\boldsymbol{H}(k)\}$.

\section{Summary of Interpolants and Factorizability}

It is well known that noncyclic FIR PU systems can be factored [23] in terms of the building blocks $\boldsymbol{I}-\boldsymbol{u}_{i} \boldsymbol{u}_{i}^{\dagger}+$ $z^{-1} \boldsymbol{u}_{i} \boldsymbol{u}_{i}^{\dagger}$, where $\boldsymbol{u}_{i}^{\dagger} \boldsymbol{u}_{i}=1$. However, in the cyclic case, factorization in terms of $\left(\boldsymbol{I}-\boldsymbol{u}_{i} \boldsymbol{u}_{i}^{\dagger}+W_{L}^{k} \boldsymbol{u}_{i} \boldsymbol{u}_{i}^{\dagger}\right)$ is not always possible. In fact, Theorem 2 shows that such factorization is possible if and only if there exists an FIR PU interpolant. However, the fact that there always exists a rational IIR interpolant (Theorem 3) means, in particular, that we can obtain a factorization of the cyclic system $\boldsymbol{H}(k)$ in terms of slightly modified building blocks. These have the form $U_{k}(z)$ and $D_{k}(z)$ discussed above, with $z^{-1}$ replaced by $W_{L}^{k}$ everywhere.

\section{State-Space Descriptions FOR CyClic LTI Systems}

In Section II-A, we considered cyclic difference equations and recursive structures for cyclic transfer functions. Statespace descriptions allow us to generalize these ideas. From the direct-form structure of Fig. 6, we can identify a set of $N$ state variables $v_{i}(n)$ (outputs of the unit delay elements $W_{L}^{k}$ ) and obtain equations of the form

$$
\begin{aligned}
\boldsymbol{v}(n+1) & =\boldsymbol{A} \boldsymbol{v}(n)+\boldsymbol{B} \boldsymbol{x}(n) \\
\boldsymbol{y}(n) & =\boldsymbol{C} \boldsymbol{v}(n)+\boldsymbol{D} \boldsymbol{x}(n)
\end{aligned}
$$

where $\boldsymbol{v}(n)$ is the state vector given by

$$
\boldsymbol{v}(n)=\left[\begin{array}{llll}
v_{1}(n) & v_{2}(n) & \cdots & v_{N}(n)
\end{array}\right]^{T} .
$$

More generally, given any structure for a cyclic LTI system, we can identify the outputs of the cyclic delay elements $W_{L}^{k}$ as the state variables and obtain the above equations. Since this system can have multiple inputs and outputs, we have used bold letters $\boldsymbol{x}(n)$ and $\boldsymbol{y}(n)$ above. Repeated use of the first equation in (35) yields $\boldsymbol{v}(L)=\boldsymbol{A}^{L} \boldsymbol{v}(0)+$, a linear combination of samples of $\boldsymbol{x}(n)$. Since all the time indices are interpreted modulo- $L$, we have $v(L)=v(0)$, and

$$
\left(\boldsymbol{I}-\boldsymbol{A}^{L}\right) \boldsymbol{v}(0)=\text { linear combination of samples of } \boldsymbol{x}(n) .
$$

Thus, we can identify the initial state $v(0)$, provided $I-A^{L}$ is nonsingular, i.e., no eigenvalue of $\boldsymbol{A}$ has the form $W_{L}^{m}$ for any integer $m$. In other words, the eigenvalues of $\boldsymbol{A}$ should not be at the unit-circle points indicated in Fig. 2. This nonsingularity condition can be understood in another way. If we evaluate the frequency response $\boldsymbol{H}(k)$ explicitly, we would have the form

$$
\boldsymbol{H}(k)=\boldsymbol{D}+\boldsymbol{C}\left(W_{L}^{-k} \boldsymbol{I}-\boldsymbol{A}\right)^{-1} \boldsymbol{B}=\frac{P(k)}{\operatorname{det}\left(W_{L}^{-k} \boldsymbol{I}-A\right)} .
$$

The eigenvalue condition on $\boldsymbol{A}$ implies that the preceding denominator $\operatorname{det}\left(W_{L}^{-k} I-A\right)$ is nonzero for all integers $k$. As long as the eigenvalue condition is satisfied, $\boldsymbol{H}(k)$ is defined for all $k$, and we can uniquely identify an "initial state" $\boldsymbol{v}(0)$ for any input sequence $\{\boldsymbol{x}(n)\}$.

Even though the expression for $\boldsymbol{H}(k)$ resembles the noncyclic case $H_{\text {non }}(z)=D+C(z \boldsymbol{I}-\boldsymbol{A})^{-1} \boldsymbol{B}$, the impulse 
response matrix $\boldsymbol{h}(n)$ takes a slightly different form as compared with the noncyclic case [23]

$$
\boldsymbol{h}(n)= \begin{cases}\boldsymbol{D}+\boldsymbol{C} \boldsymbol{A}^{L-1}\left(\boldsymbol{I}-\boldsymbol{A}^{L}\right)^{-1} \boldsymbol{B}, & n=0 \\ \boldsymbol{C} \boldsymbol{A}^{n-1}\left(\boldsymbol{I}-\boldsymbol{A}^{L}\right)^{-1} \boldsymbol{B}, & 1 \leq n \leq L-1\end{cases}
$$

Notice, for example, that $\boldsymbol{h}(0) \neq \boldsymbol{D}$, which is a departure from the noncyclic case. These differences arise because the initial condition $v(0)$ is predetermined, as explained earlier, and cannot be set to zero (as we would in the noncyclic case). We can verify by explicit computation that the expressions for $\boldsymbol{h}(n)$ and $\boldsymbol{H}(k)$ are indeed related as $\boldsymbol{H}(k)=\sum_{n=0}^{L-1} \boldsymbol{h}(n) W_{L}^{n k}$. As a final remark, we mention that although the computation of $\boldsymbol{v}(0)$, in general, requires an initial overhead, such a computation followed by the recursive computation of $y(n)$ as in (35) is often more economic than direct or FFT-based circular convolution of $\boldsymbol{x}(n)$ and $\boldsymbol{h}(n)$.

Similarity Transformations: If we define a new state-space description by using the familiar similarity transformation $A_{1}=T^{-1} A \boldsymbol{T}, B_{1}=T^{-1} B, C_{1}=C \boldsymbol{T}$, we can verify that the new system $\left(\boldsymbol{A}_{1}, \boldsymbol{B}_{1}, \boldsymbol{C}_{1}, \boldsymbol{D}\right)$ has the same $h(n)$. The reason for this is that we can verify by substitution that $\boldsymbol{C} A^{n-1}\left(\boldsymbol{I}-\boldsymbol{A}^{L}\right)^{-1} \boldsymbol{B}$ is unchanged by the similarity transform for any $n \geq 1$. Thus, we can find equivalent cyclic statespace realizations by using similarity transforms. Note that even though $\boldsymbol{D}$ does not represent $\boldsymbol{h}(0)$, it is still unchanged in the similarity transformation.

\section{A. Reachability and Observability}

The ideas of reachability and observability [6], [9], [23] can be extended to cyclic LTI systems, but there are some differences from the traditional noncyclic case. For example, we will see that reachability and observability together do not imply minimality. The cyclic LTI system is said to be reachable if we can arrive at any chosen final value $\boldsymbol{v}_{f}$ for the state vector $\boldsymbol{v}(n)$ at any chosen time $n$ by proper choice of the input sequence $\boldsymbol{x}(\cdot)$. To quantify this, consider the state recursion $\boldsymbol{v}(n+1)=\boldsymbol{A} \boldsymbol{v}(n)+\boldsymbol{B} \boldsymbol{x}(n)$ again. If we apply this $L$ times and use the periodicity conditions $\boldsymbol{v}(n+L)=\boldsymbol{v}(n)$ and $\boldsymbol{x}(n+L)=\boldsymbol{x}(n)$, we find

$$
\left(\boldsymbol{I}-A^{L}\right) \boldsymbol{v}(n)=\underbrace{\left[\begin{array}{llll}
\boldsymbol{B} & \boldsymbol{A} B & \cdots & \boldsymbol{A}^{L-1} \boldsymbol{B}
\end{array}\right]}_{\mathcal{R}_{A, B}(L)}\left[\begin{array}{c}
\boldsymbol{x}(n-1) \\
\boldsymbol{x}(n-2) \\
\vdots \\
\boldsymbol{x}(n-L)
\end{array}\right] .
$$

Here, we have used the notation that for any positive integer $i$

$$
\mathcal{R}_{A, B}(i) \triangleq\left[\begin{array}{llll}
B & A B & \cdots & A^{i-1} B
\end{array}\right] .
$$

Let $N$ denote the state dimension (size of $\boldsymbol{v}(n)$ ) and $r$ the number of inputs (size of $\boldsymbol{x}(n)$ ). Then, $\mathcal{R}_{A, B}(i)$ is a $N \times i r$ matrix with rank $\leq N$. The matrix $\mathcal{R}_{A, B}(L)$, in particular, has size $N \times L r$. Assume that $\left(\boldsymbol{I}-\boldsymbol{A}^{L}\right)$ is nonsingular for reasons explained earlier. It is then clear that we can attain any value for the state $\boldsymbol{v}(n)$ at any time $n$ by application of a suitable input $\boldsymbol{x}(n-1), \boldsymbol{x}(n-2), \cdots, \boldsymbol{x}(n-L)$ if and only if the matrix
$\mathcal{R}_{A, B}(L)$ has rank $N$. This gives a test for reachability. Now, two cases should be distinguished.

1) Let $N \leq L$. Then, the rank of $\mathcal{R}_{A, B}(N)$ will be equal to that of $\mathcal{R}_{A, B}(L)$ (Cayley-Hamilton theorem). Then, the reachability test reduces to the conventional one. Moreover, in the nonreachable case, we can perform the usual reduction, and reduce the size $N$ of the state vector.

2) Let $N>L$. This is possible in the mimo case (e.g., if $\boldsymbol{H}(k)=W_{L}^{k} \boldsymbol{I}_{r}$, then $N=r$, regardless of $\left.L\right)$. For this case, two subcases are possible.

a) The rank of $\mathcal{R}_{A, B}(L)$ is already $N$; therefore, the system is reachable.

b) The rank of $\mathcal{R}_{A, B}(L)$ is smaller than that of $\mathcal{R}_{A, B}(N)$. If the latter is also less than $N$, we can perform the usual reduction and reduce the size $N$ of the state vector. If the rank of $\mathcal{R}_{A, B}(N)$ is already $N$, we cannot do this, but we might still be able to perform a reduction of the cyclic state-space equations, as we shall demonstrate below.

State observability in a cyclic LTI system can also be defined similar to the traditional case but with some subtle distinctions between the cases $N \leq L$ and $N>L$. First, assume $N \leq L$. The output equation $\boldsymbol{y}(n)=\boldsymbol{C} \boldsymbol{v}(n)+\boldsymbol{D} \boldsymbol{x}(n)$ can be repeatedly applied to yield

$$
\begin{aligned}
& {\left[\begin{array}{c}
\boldsymbol{y}(n) \\
\boldsymbol{y}(n+1) \\
\vdots \\
\boldsymbol{y}(n+N-1)
\end{array}\right]} \\
& =\left[\begin{array}{c}
C \\
C A \\
\vdots \\
\boldsymbol{C A}^{N-1}
\end{array}\right] \\
& \mathcal{S}_{C, A}(N)
\end{aligned}
$$

The initial state $v(n)$ can be uniquely found from the $N$ samples of the input and output in this equation, as long as the matrix $\mathcal{S}_{C, A}(N)$, which has $N$ columns, has rank $N$. If $N>L$, the preceding equation is not meaningful because $y(i)$ and $\boldsymbol{x}(i)$ repeat with period $L$. In this case, however, we have a very unusual situation. If the input and output are known for all $L$ values of time, then in particular, $\boldsymbol{x}(i)$ is known for all $i$, and we can identify the state $\boldsymbol{v}(n)$ for all $n$ using the state recursion. Thus, the notion of observability becomes trivial for $N>L$.

Example 2: Consider the cyclic system

$$
H(k)=1+a W_{L}^{k}+a^{2} W_{L}^{2 k}+\cdots+a^{L-1} W_{L}^{(L-1) k}
$$

for which a direct-form implementation is shown in Fig. 14(a). With state variables as indicated, the state-space description 


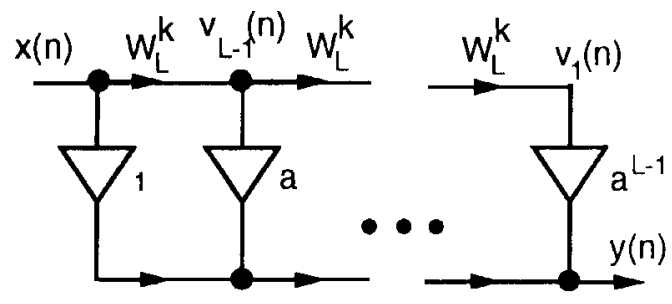

(a)

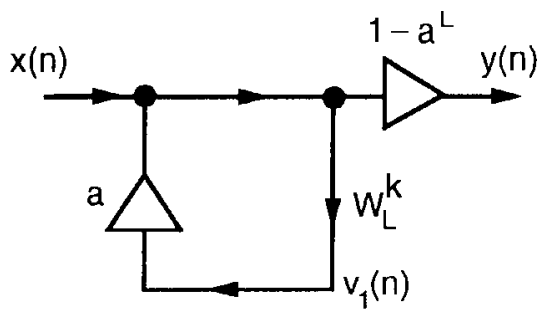

(b)

Fig. 14. Example 2. Two implementations of a $\operatorname{cyclic}(L)$ system. Both of these are reachable and observable implementations. (a) $L-1$ state variables used. (b) Only one state variable used.

$(A, B, C, D)$ can readily be identified, yielding

$$
\begin{aligned}
\boldsymbol{A} & =\left[\begin{array}{ccccc}
0 & 1 & 0 & \cdots & 0 \\
0 & 0 & 1 & \cdots & 0 \\
\vdots & \vdots & \vdots & \ddots & \vdots \\
0 & 0 & 0 & \cdots & 1 \\
0 & 0 & 0 & \cdots & 0
\end{array}\right], \quad B=\left[\begin{array}{c}
0 \\
0 \\
\vdots \\
0 \\
1
\end{array}\right] \\
\boldsymbol{C} & =\left[\begin{array}{lllll}
a^{L-1} & a^{L-2} & \cdots & a
\end{array}\right] \quad D=1 .
\end{aligned}
$$

Note that the number of state variables $N=L-1$. From the preceding, we verify that

$$
\begin{aligned}
\mathcal{R}_{A, B}(L) & =\left[\begin{array}{cccccc}
0 & 0 & \cdots & 0 & 1 & 0 \\
0 & 0 & \cdots & 1 & 0 & 0 \\
\vdots & \vdots & \ddots & \vdots & \vdots & \vdots \\
0 & 1 & \cdots & 0 & 0 & 0 \\
1 & 0 & \cdots & 0 & 0 & 0
\end{array}\right] \\
\mathcal{S}_{C, A}(N) & =\left[\begin{array}{cccc}
a^{L-1} & a^{L-2} & \cdots & a \\
0 & a^{L-1} & \cdots & a^{2} \\
\vdots & \vdots & \ddots & \vdots \\
0 & 0 & \cdots & a^{L-1}
\end{array}\right] .
\end{aligned}
$$

Since $N=L-1, \mathcal{R}_{A, B}(L)$ has size $(L-1) \times L$, and $\mathcal{S}_{C, A}(N)$ has size $(L-1) \times(L-1)$. Both of these matrices have rank $N=L-1$ (assuming, of course, $a \neq 0$ ), showing that the structure is both reachable and observable. Notice, however, that the system $H(k)$ can be rewritten in the recursive form

$$
H(k)=\frac{1-a^{L}}{1-a W_{L}^{k}}
$$

using the fact that $W_{L}^{L}=1$. This yields the simpler recursive implementation requiring only one cyclic delay $W_{L}^{k}$ [Fig. 14(b)]. We can verify that the state space description of the simplified structure is

$$
A=a, \quad B=1, \quad C=a\left(1-a^{L}\right), \quad D=1-a^{L} .
$$

In this case, the number of state variables $N=1$. We readily verify that $\mathcal{R}_{A, B}(1)=1$ and $\mathcal{S}_{C, A}(1)=a\left(1-a^{L}\right)$. Therefore, $\mathcal{R}_{A, B}(L)$ and $\mathcal{S}_{C, A}(N)$ have rank $N$, and the structure is reachable and observable (assuming $a \neq 0$ and $a^{L} \neq 1$ ). Thus the two structures shown in Fig. 14 are two reachable and observable implementations of $H(k)$ with different state dimensions. The first one requires $L-1$ cyclic delays (the $W_{L}^{k}$ elements), whereas the second structure requires only one cyclic delay. Notice also that the quantity $D$ is different for the two structures, unlike noncyclic systems. This is consistent with the fact that for cyclic systems $h(0) \neq D$ but is given by the more elaborate expression (39).

Example 3: Consider the $2 \times 2$ cyclic system shown in Fig. 15(a), and assume $L=3$. The number of state variables is $N=4$. The state space description has

$$
\begin{aligned}
\boldsymbol{A} & =\left[\begin{array}{llll}
0 & 1 & 0 & 0 \\
0 & 0 & 1 & 0 \\
0 & 0 & 0 & 1 \\
0 & 0 & 0 & 0
\end{array}\right], \quad \boldsymbol{B}=\left[\begin{array}{ll}
0 & 0 \\
1 & 1 \\
0 & 0 \\
1 & 1
\end{array}\right] \\
\boldsymbol{C} & =\left[\begin{array}{llll}
0 & 0 & 1 & 0 \\
1 & 0 & 0 & 0
\end{array}\right] .
\end{aligned}
$$

Then, explicit computation shows that

$$
\begin{aligned}
\mathcal{R}_{A, B}(L) & =\left[\begin{array}{llllll}
0 & 0 & 1 & 1 & 0 & 0 \\
1 & 1 & 0 & 0 & 1 & 1 \\
0 & 0 & 1 & 1 & 0 & 0 \\
1 & 1 & 0 & 0 & 0 & 0
\end{array}\right] \\
\mathcal{R}_{A, B}(N) & =\left[\begin{array}{llllllll}
0 & 0 & 1 & 1 & 0 & 0 & 1 & 1 \\
1 & 1 & 0 & 0 & 1 & 1 & 0 & 0 \\
0 & 0 & 1 & 1 & 0 & 0 & 0 & 0 \\
1 & 1 & 0 & 0 & 0 & 0 & 0 & 0
\end{array}\right] \\
\mathcal{S}_{C, A}(2) & =\left[\begin{array}{llll}
0 & 0 & 1 & 0 \\
1 & 0 & 0 & 0 \\
0 & 0 & 0 & 1 \\
0 & 1 & 0 & 0
\end{array}\right] .
\end{aligned}
$$

Thus, $\mathcal{R}_{A, B}(L)$ has rank $3<N$, which shows that the cyclic system is not reachable. However, $\mathcal{R}_{A, B}(N)$ has rank 4. Since $\mathcal{S}_{C, A}(2)$ has rank 4, so does $\mathcal{S}_{C, A}(N)$. Therefore, we cannot perform state reduction using classical techniques. In this example, however, it is possible to perform state reduction of the cyclic system by simple manipulations of the structure and by using the fact that $W_{L}^{3}=1$. For this, we notice the identity

$$
\left[\begin{array}{ll}
1 & 1 \\
1 & 1
\end{array}\right]\left[\begin{array}{ll}
1 & 0 \\
0 & x
\end{array}\right]\left[\begin{array}{ll}
1 & 1 \\
1 & 1
\end{array}\right]=(1+x)\left[\begin{array}{ll}
1 & 1 \\
1 & 1
\end{array}\right]
$$




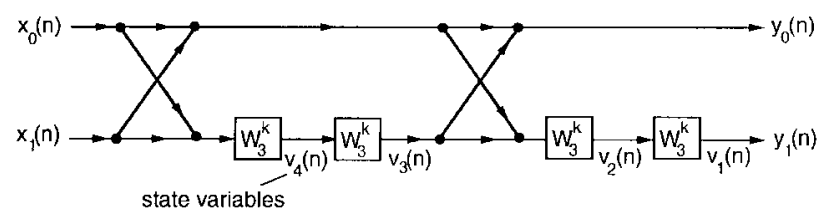

(a)

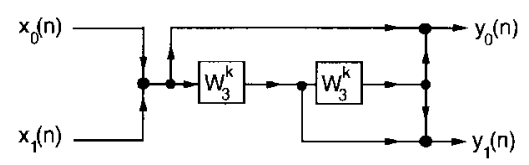

(b)

Fig. 15. Example 3. (a) Cyclic(3) system requiring four cyclic delays. (b) Reduced system requiring two cyclic delays. Classical techniques cannot be used to perform this reduction.

which shows that the transfer matrix of Fig. 15(a) is eventually

$$
\begin{aligned}
\boldsymbol{H}(k) & =\left[\begin{array}{cc}
1+W_{3}^{2 k} & 0 \\
0 & W_{3}^{2 k}+W_{3}^{k}
\end{array}\right]\left[\begin{array}{ll}
1 & 1 \\
1 & 1
\end{array}\right] \\
& =\left[\begin{array}{c}
1+W_{3}^{2 k} \\
W_{3}^{k}+W_{3}^{2 k}
\end{array}\right]\left[\begin{array}{ll}
1 & 1
\end{array}\right]
\end{aligned}
$$

which has the implementation shown in Fig. 15(b) requiring only two cyclic delays. Thus, in this example, $\mathcal{R}_{A, B}(N)$ and $\mathcal{S}_{C, A}(N)$ have rank $N$, but $\mathcal{R}_{A, B}(L)$ does not, and we have been able to reduce the state dimension.

In Example 2, we found that the state dimension could be reduced, even though the cyclic system is reachable as well as observable. In Example 3 , we found that $\mathcal{R}_{A, B}(N)$ and $\mathcal{S}_{C, A}(N)$ have rank $N$ and $\mathcal{R}_{A, B}(L)$ has deficient rank, and the state dimension could again be reduced. The question now is, what is a necessary and sufficient condition for the minimality of state dimension in cyclic LTI structures? A related question is, can we develop a theory paralleling the Smith-McMillan form and relate the minimum state dimension (McMillan degree) to this form? These appear to be fundamental questions requiring future work.

\section{B. Unitariness of Realization Matrix}

Suppose we are given an implementation for a cyclic transfer matrix $\boldsymbol{E}(k)$. This implementation has a state-space description of the form (35). The realization matrix for the implementation is defined as

$$
\left[\begin{array}{ll}
A & B \\
C & D
\end{array}\right]
$$

The following result connects the cyclic-PU property to unitariness of the realization matrix.

Lemma 1: If the realization matrix is unitary, then the cyclic system $\boldsymbol{E}(k)$ is PU.

Proof: Rewrite the state equations as

$$
\left[\begin{array}{c}
\boldsymbol{v}(n+1) \\
\boldsymbol{y}(n)
\end{array}\right]=\left[\begin{array}{ll}
A & B \\
\boldsymbol{C} & \boldsymbol{D}
\end{array}\right]\left[\begin{array}{l}
\boldsymbol{v}(n) \\
\boldsymbol{x}(n)
\end{array}\right]
$$

Unitariness of the realization matrix implies $\|\boldsymbol{v}(n+1)\|^{2}+$ $\|\boldsymbol{y}(n)\|^{2}=\|\boldsymbol{v}(n)\|^{2}+\|\boldsymbol{x}(n)\|^{2}$, where $\|\boldsymbol{v}\|^{2}$ denotes $\boldsymbol{v}^{\dagger} \boldsymbol{v}$. If we write the preceding equation for $0 \leq n \leq L-1$ and add them up, we obtain

$$
\sum_{n=0}^{L-1} \boldsymbol{y}^{\dagger}(n) \boldsymbol{y}(n)=\sum_{n=0}^{L-1} \boldsymbol{x}^{\dagger}(n) \boldsymbol{x}(n)
$$

by using the fact that $\boldsymbol{v}(n+L)=\boldsymbol{v}(n)$. With $X(k)=$ $\Sigma_{n=0}^{L-1} \boldsymbol{x}(n) W_{L}^{n k}$ and $\boldsymbol{Y}(k)=\Sigma_{n=0}^{L-1} \boldsymbol{y}(n) W_{L}^{n k}$, we then obtain (using Parseval's relation) $\sum_{k=0}^{L-1} \quad \boldsymbol{Y}^{\dagger}(k) \boldsymbol{Y}(k)=$ $\sum_{k=0}^{L-1} \boldsymbol{X}^{\dagger}(k) \boldsymbol{X}(k)$, that is

$$
\sum_{k=0}^{L-1} \boldsymbol{X}^{\dagger}(k) \boldsymbol{E}^{\dagger}(k) \boldsymbol{E}(k) \boldsymbol{X}(k)=\sum_{k=0}^{L-1} \boldsymbol{X}^{\dagger}(k) \boldsymbol{X}(k) .
$$

This should hold for all sequences $\{\boldsymbol{X}(k)\}$, which implies that $\boldsymbol{X}^{\dagger}(k) \boldsymbol{E}^{\dagger}(k) \boldsymbol{E}(k) \boldsymbol{X}(k)=\boldsymbol{X}^{\dagger}(k) \boldsymbol{X}(k)$ for any $\boldsymbol{X}(k)$, proving $\boldsymbol{E}^{\dagger}(k) \boldsymbol{E}(k)=\boldsymbol{I}$ indeed. $\quad \nabla \nabla \nabla$

This result is analogous to a result in the noncyclic case [23]. However, unlike in the noncyclic case, we do not have the converse result. That is, even if $\boldsymbol{E}(k)$ is PU, there may not exist a minimal nonrecursive structure (i.e., minimal structure with all eigenvalues of $\boldsymbol{A}$ equal to zero) with unitary system matrix. When such a structure does exist, the FIR interpolant $\boldsymbol{E}_{\text {int }}(z)=\boldsymbol{D}+\boldsymbol{C}(z \boldsymbol{I}-\boldsymbol{A})^{-1} \boldsymbol{B}$ obtained by replacing $W_{L}^{k}$ with $z^{-1}$ in the structure would be PU (because a result like Lemma 1 also holds in the noncyclic case [23]). Since FIR PU interpolants do not always exist (Section V-B), the point is proved. By combining this observation and Theorem 2, we obtain the following result.

Theorem 4: Let $\boldsymbol{E}(k)$ be cyclic-PU. Then, the following statements are equivalent.

1) There exists a causal FIR PU interpolant $\boldsymbol{E}_{\text {int }}(z)$.

2) $\boldsymbol{E}(k)$ can be factorized into unitary building blocks as in (33).

3) There exists a cyclic implementation of $\boldsymbol{E}(k)$ such that the realization matrix is unitary.

\section{CONCLUDING REMARKS}

The main purpose of this paper has been to introduce the idea of cyclic LTI systems, place in evidence some interesting theoretical properties, and point out a few open problems. The emphasis has primarily been on cyclic versions of recursive difference equations, allpass filters, paraunitary matrices, multirate filter banks, and state space theory. It will be interesting to figure out how to exploit the extra freedom offered by the cyclic system, e.g., for the design of subband coders. Can this be exploited to obtain increased coding gain (or compression) or to reduce the complexity of implementations? Evidently, more work is necessary in order to assess the practical advantages. We saw that cyclic LTI systems open up interesting problems in the more general area of signal and system theory. Many of these were mentioned throughout the paper. We can also attempt to formulate cyclic versions of other standard problems in filter bank theory, for example, cosine modulated filter bank design. The important thing again would be to establish a clear advantage for such extensions. 


\section{APPENDIX A}

\section{Proof of CyClic-Decimation Formula}

By definition, the output of the cyclic decimator is

$$
\begin{aligned}
y(n) & =x(M n)=\frac{1}{L} \sum_{m=0}^{L-1} X(m) W_{L}^{-m(M n)} \\
& =\frac{1}{L} \sum_{m=0}^{L-1} X(m) W_{K}^{-m n} \\
& =\frac{1}{L} \sum_{k=0}^{K-1} W_{K}^{-k n} \sum_{i=0}^{M-1} X(K i+k) \\
& =\frac{1}{K} \sum_{k=0}^{K-1} W_{K}^{-k n} \frac{1}{M} \sum_{i=0}^{M-1} X(K i+k) .
\end{aligned}
$$

Therefore, the $K$-point DFT of $y(n)$ is $\sum_{i=0}^{M-1} X(K i+$ $k) / M$, or equivalently, $\Sigma_{i=0}^{M-1} X(k-i K) / M$.

\section{APPENDIX B \\ CYCLIC Alias-COMPONENT MatRIX}

Using the decimator and expander formulas (7) and (9), we can express the $L$-point DFT of the reconstructed signal $\hat{x}(n)$ as

$$
\hat{X}(k)=\sum_{i=0}^{M-1} X(k-i K) \sum_{m=0}^{M-1} \frac{1}{M} H_{m}(k-i K) F_{m}(k)
$$

where $K=L / M$. The alias components are $X(k-i K), i \neq 0$, and the desired signal term is $X(k)$. Therefore, the perfect reconstruction condition is

$$
\boldsymbol{H}(k)\left[\begin{array}{c}
F_{0}(k) \\
F_{1}(k) \\
\vdots \\
F_{M-1}(k)
\end{array}\right]=\left[\begin{array}{c}
M \\
0 \\
\vdots \\
0
\end{array}\right]
$$

where $\boldsymbol{H}(k)$ is the alias component (AC) matrix with $(i, m)$ element $[\boldsymbol{H}(k)]_{i m}=H_{m}(k-i K)$. Now, the analysis filters and polyphase matrix are related as

$$
\begin{aligned}
& {\left[\begin{array}{llll}
H_{0}(k) & H_{1}(k) & \cdots & H_{M-1}(k)
\end{array}\right]} \\
& \quad=\left[\begin{array}{llll}
1 & W_{L}^{k} & \cdots & W_{L}^{(M-1) k}
\end{array}\right] \boldsymbol{E}^{T}(k) .
\end{aligned}
$$

Using the fact that $\boldsymbol{E}(k)$ is periodic $(K)$, we can show that $\boldsymbol{H}(k)=\boldsymbol{W}_{M}^{*} \Lambda(k) \boldsymbol{E}^{T}(k)$, where $W_{M}$ is the $M \times M$ DFT matrix, and $\boldsymbol{A}(k)$ is diagonal with $i$ th element $W_{L}^{i k}$. Thus, orthonormality (unitarity of $\boldsymbol{E}(k)$ ) is equivalent to $\boldsymbol{H}^{\dagger}(k) \boldsymbol{H}(k)=M \boldsymbol{I}$. In the two-channel case, the cyclic AC matrix is

$$
\left[\begin{array}{cc}
H_{0}(k) & H_{1}(k) \\
H_{0}(k-K) & H_{1}(k-K)
\end{array}\right]
$$

where $K=L / 2$. If $H_{0}(k)$ satisfies the cyclic power symmetry property $\left|H_{0}(k)\right|^{2}+\left|H_{0}(k-K)\right|^{2}=2$ and if $H_{1}(k)=-W_{L}^{k} H_{0}^{*}(k-K)$, we can verify by substitution that $\boldsymbol{H}^{\dagger}(k) \boldsymbol{H}(k)=2 \boldsymbol{I}$, and the cyclic filter bank is indeed orthonormal. Finally, the theory of alias-free filter banks (i.e., the pseudocirculant conditions [23]) can be extended to the cyclic case in a straightforward manner; therefore, we skip the details.

Cyclic Orthonormal Basis: For orthonormal cyclic filter banks with perfect reconstruction, $\boldsymbol{R}(k)$ is also unitary; therefore, the matrix $\boldsymbol{F}(k)$ whose elements are $[\boldsymbol{F}(k)]_{r m}=$ $F_{m}(k-r K)$ also satisfies $\boldsymbol{F}^{\dagger}(k) \boldsymbol{F}(k)=M \boldsymbol{I}$, that is, $\sum_{r=0}^{M-1} F_{i}(k-r K) F_{m}^{*}(k-r K) / M=\delta(i-m)$. The left-hand side is the $K$-point DFT of the $M$-fold decimated version of $g(\ell) \triangleq \Sigma_{n=0}^{L-1} f_{i}(n) f_{m}^{*}(n-\ell)$. Thus, orthonormality of $\boldsymbol{R}(k)$ is equivalent to $\sum_{n=0}^{L-1} f_{i}(n) f_{m}^{*}(n-M \ell)=\delta(i-m) \delta(\ell)$, which proves (19).

\section{APPENDIX C}

\section{PROOF OF THEOREM 1}

The FIR matrix $\boldsymbol{H}(z)$ is of the form

$$
\boldsymbol{H}(z)=\left[\begin{array}{ll}
H_{0}(z) & G_{0}(z) \\
H_{1}(z) & G_{1}(z)
\end{array}\right] .
$$

Since the "if" part is obvious, we concentrate on the "only if" part. The paraunitary property $\tilde{H}(z) H(z)=\boldsymbol{I}$ implies, among other things, that

$$
\begin{aligned}
\tilde{H}_{0}(z) H_{0}(z)+\tilde{H}_{1}(z) H_{1}(z) & =1 \\
\tilde{H}_{0}(z) G_{0}(z)+\tilde{H}_{1}(z) G_{1}(z) & =0 .
\end{aligned}
$$

Suppose an element in $\boldsymbol{H}(z)$ is identically zero, e.g., $H_{1}(z)=0$ for all $z$. Then, $\tilde{H}_{0}(z) G_{0}(z)=0$ for all $z$, from (52). Since $H_{0}(z)$ is nonzero from (51), we conclude that $G_{0}(z)=0$ for all $z$. Thus, the FIR paraunitary matrix $\boldsymbol{H}(z)$ becomes diagonal, proving the desired form (30). Therefore, we assume that none of the four elements in $\boldsymbol{H}(z)$ is identically zero. Now, any pair of power complementary transfer functions [i.e., a pair $H_{0}(z), H_{1}(z)$ satisfying (51)] should be free from nontrivial common factors (i.e., factors other than $c z^{-n_{0}}$ ) because the left side of (51) should be nonzero for all $z$. Therefore, $H_{0}(z)$ and $H_{1}(z)$ have no nontrivial common factors. The same holds for the pair $G_{0}(z)$ and $G_{1}(z)$ and the pair $\tilde{H}_{0}(z)$ and $\tilde{H}_{1}(z)$. Therefore, from (52), we conclude that

$$
G_{0}(z)=c_{0} z^{-n_{0}} \tilde{H}_{1}(z), \quad G_{1}(z)=c_{1} z^{-n_{1}} \tilde{H}_{0}(z)
$$

which upon substitution into (52) yields $\left(c_{0} z^{-n_{0}}+\right.$ $\left.c_{1} z^{-n_{1}}\right) \tilde{H}_{0}(z) \tilde{H}_{1}(z)=0$, proving $c_{0} z^{-n_{0}}+c_{1} z^{-n_{1}}=0$. Thus, $c_{0}=-c_{1}$, and $n_{0}=n_{1}$. Since the row elements $H_{0}(z)$ and $G_{0}(z)$ are also power complementary, i.e., $\quad \tilde{H}_{0}(z) H_{0}(z)+\tilde{G}_{0}(z) G_{0}(z)=1$, we get $\tilde{H}_{0}(z) H_{0}(z)+\left|c_{0}\right|^{2} \tilde{H}_{1}(z) H_{1}(z)=1$. Comparing this with (51), we conclude $\left|c_{0}\right|^{2}=1$, i.e., $c_{0}=e^{j \theta}$. This establishes the form (30).

\section{REFERENCES}

[1] A. N. Akansu and R. A. Haddad, Multiresolution Signal Decomposition: Transforms, Subbands, and Wavelets. New York: Academic, 1992.

[2] A. Antoniou, Digital Filters: Analysis and Design. New York; McGraw-Hill, 1979.

[3] A. S. Bopardikar, M. R. Raghuveer, and B. S. Adiga, "Perfect reconstruction circular convolution filter banks and their applications to the implementation of bandlimited discrete wavelet transforms," in Proc. 
IEEE Int. Conf. Acoust., Speech, Signal Process., Munich, Germany, May 1997, pp. 3665-3668.

[4] A. S. Bopardikar, M. R. Raghuveer, and B. S. Adiga, "PRCC filter banks: Theory, implementation, and application," Proc. SPIE, San Diego, CA, July-Aug. 1997, vol. 3169.

[5] G. Caire, R. L. Grossman, and H. V. Poor, "Wavelet transforms associated with finite cyclic groups," IEEE Trans. Inform. Theory, vol. 39 pp. $1157-1166$, July 1993.

[6] C. T. Chen, Linear System Theory and Design. New York: Holt, Rinehart, and Winston, 1984.

[7] R. L. de Queiroz and K. R. Rao, "On reconstruction methods for processing finite-length signals with paraunitary filter banks," IEEE Trans. Signal Processing, vol. 43, pp. 2407-2410, Oct. 1995.

[8] B. Gold and C. M. Rader, Digital Processing of Signals. New York: McGraw-Hill, 1969.

[9] T. Kailath, Linear Systems. Englewood Cliffs, NJ: Prentice-Hall, 1980.

[10] G. Karlsson and M. Vetterli, "Extension of finite length signals for subband coding," Signal Process., vol. 17, pp. 161-168, June 1989.

[11] H. Kiya, K. Nishikawa, and M. Sagawa, "Property of circular convolution for subband image coding," IEICE Trans. Fundamentals, vol. E75-A, no. 7, pp. 852-860, July 1992.

[12] H. S. Malvar, Signal Processing with Lapped Transforms. Norwood, MA: Artech House, 1992.

[13] R. Motwani and K. R. Ramakrishnan, "Design of two channel linear phase orthogonal cyclic filter banks," IEEE Signal Processing Lett., vol 5, pp. 121-123, May 1998.

[14] K. Nishikawa, K. Miyazaki, and H. Kiya, "A parallel AR spectral estimation using a new class of filter bank," Proc. IEEE Int. Conf. Acoust., Speech, Signal Process., Apr. 1993, pp. 244-247.

[15] V. Nuri and R. H. Bamberger, "Size-limited filter banks for subband image compression," IEEE Trans. Image Processing, vol. 7, pp. 1317-1323, Sept. 1995.

[16] H. J. Nussbaumer, "Digital filtering using polynomial transforms," Electron. Lett., vol. 13, pp. 386-387, 1977.

[17] A. V. Oppenheim and R. W. Schafer, Discrete Time Signal Processing. Englewood Cliffs, NJ: Prentice-Hall, 1989.

[18] M. J. T. Smith and T. P. Barnwell, III, "Exact reconstruction techniques for tree-structured subband coders," IEEE Trans. Acoust., Speech, Signal Processing, vol. ASSP-34, pp. 434-441, June 1986.

[19] M. J. T. Smith and S. L. Eddins, "Analysis/synthesis techniques for subband image coding," IEEE Trans. Acoust., Speech, Signal Processing, vol. 38, pp. 1446-1456, Aug. 1990.
[20] R. A. Roberts and C. T. Mullis, Digital Signal Processing. Reading, MA: Addison-Wesley, 1987

[21] G. Strang and T. Nguyen, Wavelets and Filter Banks. Wellesley, MA: Wellesley-Cambridge, 1996.

[22] P. P. Vaidyanathan and V. C. Liu, "Efficient reconstruction of bandlimited sequences from nonuniformly decimated versions by use of polyphase filter banks," IEEE Trans. Acoust., Speech, Signal Processing, vol. 38, pp. 1927-1936, Nov. 1990.

[23] P. P. Vaidyanathan, Multirate Systems and Filter Banks. Englewood Cliffs, NJ: Prentice Hall, 1993.

[24] P. P. Vaidyanathan and A. Kirac, "Theory of cyclic filter banks," in Proc. IEEE Int. Conf. Acoust., Speech, Signal Process., Munich, Germany, May 1997, pp. 2449-2452.

[25] "Cyclic systems and the paraunitary interpolation problem," Proc. IEEE Int. Conf. Acoust., Speech, Signal Process., Seattle, WA, May 1998, pp. $1445-1448$.

[26] P. P. Vaidyanathan, "Results on cyclic signal processing systems," in Proc. EUSIPCO, Island of Rhodes, Greece, Sept. 1998.

[27] M. Vetterli and J. Kovav̌ić, Wavelets and Subband Coding. Englewood Cliffs, NJ: Prentice-Hall, 1995.

P. P. Vaidyanathan (S'80-M'83-SM'91-F'93), for photograph and biography, see this issue, p. 407.

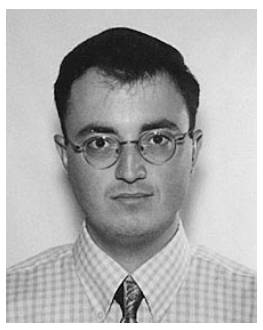

Ahmet Kiraç (S'98) was born in Afsin, Turkey, on October 10, 1971. He received the B.S. degree in electrical engineering from Bilkent University, Ankara, Turkey, in 1993 and the M.S. and Ph.D. degrees in electrical engineering from the California Institute of Technology, Pasadena, in 1994, and 1998, respectively.

He is currently with Lucent Technologies, Huntington Beach, CA. His research interests are in digital signal processing with applications in coding and communications. Optimization of filter banks for subband coding and lattice quantization with vector dithering have been his main research thrusts. 